\title{
OPEN Subunit vaccine protects against a clinical isolate of Mycobacterium avium in wild type and immunocompromised mouse models
}

Sasha E. Larsen ${ }^{1}$, Valerie A. Reese ${ }^{1}$, Tiffany Pecor ${ }^{1}$, Bryan J. Berube ${ }^{1}$, Sarah K. Cooper ${ }^{2}$, Guy Brewer ${ }^{3}$, Diane Ordway², Marcela Henao-Tamayo², Brendan K. Podell², Susan L. Baldwin ${ }^{1 \otimes}$ \& Rhea N. Coler ${ }^{1 凶}$

The nontuberculous mycobacteria (NTM) Mycobacterium avium is a clinically significant pathogen that can cause a wide range of maladies, including tuberculosis-like pulmonary disease. An immunocompromised host status, either genetically or acutely acquired, presents a large risk for progressive NTM infections. Due to this quietly emerging health threat, we evaluated the ability of a recombinant fusion protein ID91 combined with GLA-SE [glucopyranosyl lipid adjuvant, a toll like receptor 4 agonist formulated in an oil-in-water stable nano-emulsion] to confer protection in both C57BL/6 (wild type) and Beige (immunocompromised) mouse models. We optimized an aerosol challenge model using a clinical NTM isolate: $M$. avium 2-151 smt, observed bacterial growth kinetics, colony morphology, drug sensitivity and histopathology, characterized the influx of pulmonary immune cells, and confirmed the immunogenicity of ID91 in both mouse models. To determine prophylactic vaccine efficacy against this $M$. avium isolate, mice were immunized with either ID91 + GLA-SE or bacillus Calmette-Guérin (BCG). Immunocompromised Beige mice displayed a delayed influx of innate and adaptive immune cells resulting in a sustained and increased bacterial burden in the lungs and spleen compared to C57BL/6 mice. Importantly, both ID91 + GLA-SE and BCG vaccines significantly reduced pulmonary bacterial burden in both mouse strains. This work is a proofof-concept study of subunit vaccine-induced protection against NTM.

Nontuberculous mycobacteria (NTM) comprising 170 different species [and excluding Mycobacterium tuberculosis (Mtb) and M. leprae] are an emerging global health concern. Despite garnering less attention than Mtb, NTM cause a significant amount of morbidity, mortality and financial health burden in certain regions of the world, including the United States ${ }^{1}$. Indeed, exposure to NTM measured by skin sensitization testing reveals a steady increase (up to $16.6 \%$ sensitivity to $M$. intracellulare in 2000), with a corresponding decrease in skin sensitization to $\mathrm{Mtb}$ (down to $5.6 \%$ in 2000) in the United States over the same period tested ${ }^{2}$. Using death certificate reporting, researchers calculated that the age adjusted mortality rate in the United States for Mtb was 3.3 per million person years and 2.3 per million person years for NTM and rising annually in individuals not living with $\mathrm{HIV}^{3}$. These inverse trends between mycobacteria are reflected globally ${ }^{4}$. A systematic review revealed that NTM infection rates were increasing across $75 \%$ of geographic areas evaluated with $81 \%$ demonstrating declines in tuberculosis (TB) incidence ${ }^{5}$. Susceptibility to different NTM species, including M. avium, is influenced by complex host factors and the extent of pathogen exposure. The wide range of illness and disease disproportionately affects immune compromised individuals, and a suppressed immune system, either genetic or acquired, is a prominent risk factor for opportunistic NTM infections ${ }^{6-8}$.

${ }^{1}$ Center for Global Infectious Disease Research, Seattle Children's Research Institute, Seattle, WA, USA. ${ }^{2}$ Department of Microbiology, Immunology and Pathology, Colorado State University, Fort Collins, CO, USA. ${ }^{3}$ Alternative Behavior Strategies Inc, Salt Lake City, UT, USA. ${ }^{\square}$ email: susan.baldwin@seattlechildrens.org; rhea.coler@seattlechildrens.org 
NTM species are ubiquitous in the environment and can be found in soil, water or residing on fomites, which can result in repetitive host exposure ${ }^{9,10}$. Therefore, it is not surprising that the incidence of mycobacterial disease caused by NTM infections have steadily increased globally over the last 60 years ${ }^{11}$. Predominant NTM species that cause significant pulmonary disease include Mycobacterium avium complex (MAC), M. kansasii and M. abscessus ${ }^{12}$. From 2009 to 2013 approximately 61-91\% of patients evaluated for pathogenic NTM infection in the United States were confirmed to have MAC, while M. abscessus and M. kansasii ranged from 2 to $18 \%{ }^{13}$.

Despite an increased awareness of NTM infections, true incidence is likely underreported ${ }^{14-16}$. NTM disease manifestation can be remarkably similar to TB including chronic cough, lesions on chest X-ray and progressive fatigue $^{17}$, resulting in misdiagnosis. Indeed, $18 \%$ of 'chronic TB cases' in Mali were identified as NTM positive upon reevaluation by microscopy, culture and drug sensitivity testing ${ }^{18}$. Unfortunately, those patients misdiagnosed as having active Mtb infections are usually given treatments for MDR-TB. Even if properly identified, MAC is a non-reportable infectious disease in North America, which leads to great variance of incidence estimates. Centers for Disease Control and Prevention surveillance data suggests an incidence rate of 1 case per $100 \mathrm{k}$ person-years in the United States. A separate study of the United States Health Administration observed an incidence of NTM infection of 12.6 cases per $100 \mathrm{k}$ person-years in the veteran population ${ }^{19}$, suggesting a disproportionate disease burden in certain communities exist for NTM. Due to increasing prevalence, insidious virulence, and accelerating drug resistance, NTM disease is an underappreciated but growing public health burden.

Proper identification does not ensure positive prognosis, as NTM infections are notoriously difficult to treat. Current diagnostic methods are severely inadequate and clinical disease is often mistreated with ineffective drugs $^{20,21}$. Even when correctly diagnosed, a multidrug therapy is necessary for NTM treatment with contraindications for certain at risk populations ${ }^{22}$. A regimen of rifampin, ethambutol and clarithromycin or azithromycin for a period of at least 12 months following sputum culture conversion is the current recommendation for treating pulmonary MAC infection ${ }^{23}$. Historically, a meager $50-70 \%$ of individuals undergoing the correct complex multi-drug treatment regimen for chronic MAC infections reach cure ${ }^{24}$ and even then some treated patients experience relapse infections or recurrent infections with different strains of mycobacteria ${ }^{20}$. In addition, there is increasing evidence of NTM strains that are refractory to antibiotic treatment ${ }^{20,25,26}$. Novel drug therapies against MAC are being investigated for safety and efficacy ${ }^{23,27}$, but use of these new drugs may also eventually lead to the emergence of drug resistance and recurrence of disease.

The growing incidence of NTM infection combined with misdiagnosis and common treatment failure represent an expanding unmet medical need ${ }^{17}$. An alternative or complementary approach for improved therapies against NTM disease is to design, develop and evaluate vaccines against NTM. Vaccine strategies against NTM may help reduce incidence of disease and/or recurrence and could increase patient compliance by reducing the necessary duration of antibiotic treatment. Toxicity is well documented with prolonged drug regimens against NTM infections ${ }^{28}$, and therefore a shortened regimen of treatment afforded by vaccines would be of large benefit to those affected.

In this work we aimed to advance and characterize a reproducible preclinical pulmonary challenge model of a virulent strain of $M$. avium subspecies hominissuis in mice. This enabled immunogenicity and efficacy assessment using a proof-of-concept subunit vaccine candidate against $M$. avium infection in both wild type (C57BL/6) and immunocompromised (Beige) preclinical mouse models. Although a T helper $1\left(\mathrm{~T}_{\mathrm{H}} 1\right)$ immune response is partially essential for resistance to NTM infections, complete and reliable immune correlates of protection have not yet been established ${ }^{12}$. Therefore, we surveyed vaccine-induced responses that correlated with efficacy. These data are evidence that subunit vaccination against NTM species, including M. avium, is a viable mechanism to reduce this disease burden.

\section{Results}

M. avium 2-151 smt productively infects C57BL/6 mice. In order to assess the efficacy of vaccination and subsequent immune responses against NTM, we first established viable mouse models of disease. Since NTM infection and disease burden are more pronounced in immunocompromised populations, our investigation included the Beige mouse strain as the representative immunocompromised mouse model and the C57BL/6 mouse strain as a wild type (WT) resistant mouse strain. Indeed, Beige and C57BL/6 mice have been leveraged for comparative assessments of $M$. avium infections before, utilizing intranasal infections to evaluate the relative contributions of innate immune cells during early infection ${ }^{29}$. Other publications have used C57BL/6 mice with intratracheal installation ${ }^{30}$ as well as intraperitoneal injection ${ }^{31}$ to compare virulence and pathogenesis of $M$. avium isolates. A review of models for NTM therapies can be found here ${ }^{32}$. In this work we aim to expand these models using virulent $M$. avium isolates and aerosol infection routes, which we and others have used previously for mycobacterial challenge $e^{33,34}$, to evaluate vaccine-induced immunity. Interestingly, Beige mice demonstrate enhanced susceptibility to mycobacterial infections due to reduced bactericidal activity of granulocytes, a deficiency in NK cells, and a reported minor defect/delay in humoral responses ${ }^{35-37}$. We utilized the clinical isolate M. avium 2-151 smooth transparent (smt) as a challenge strain (generously provided by D. Ordway, Colorado State University) due to its enhanced virulence compared to the commonly used M. avium Chester laboratory-adapted strain, and its ability to establish a persistent pulmonary infection in the mouse model. M. avium 2-151 smt infection better represents human disease conditions from NTM infection and provides resolution for deciphering vaccine-induced protection. Indeed, while M. avium Chester bacterial burden significantly wanes over time following intranasal challenge, from the starting mean infectious challenge dose of $3.80 \log _{10}$ colony forming units (CFU) down to a mean of $2.52 \log _{10}$ CFU six weeks post infection (Fig. 1a), M. avium 2-151 smt establishes a sustained infection after intranasal challenge, with an average lung bacterial burden of $5.87 \log _{10}$ CFU four weeks post challenge, up from the challenge dose (Fig. 1a). This sustained infection of clinical MAC isolates is in agreement with published studies ${ }^{33,38,39}$ across several models as well as in our own data, whereby 
a

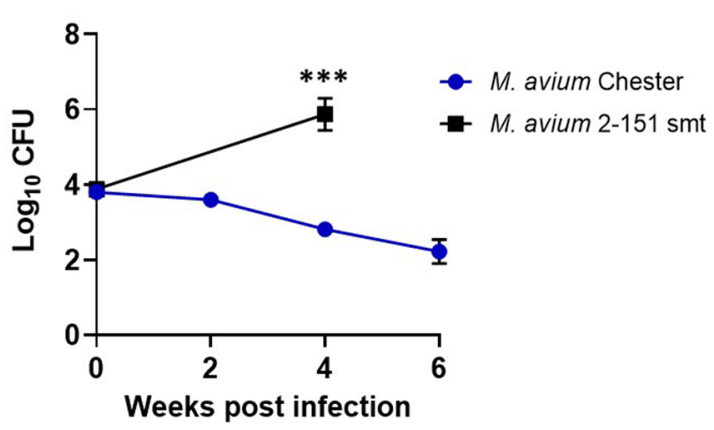

b

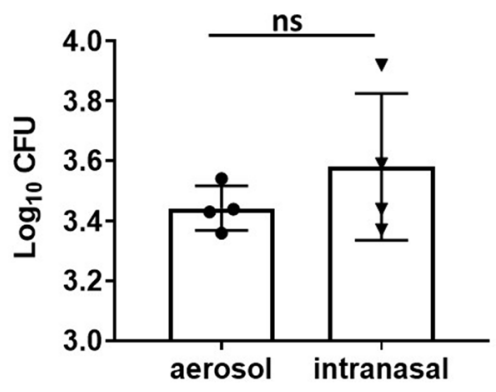

Figure 1. M. avium 2-151 smt establishes a persistent infection in C57BL/6 mice and is effectively delivered via aerosol. (a) Lung bacterial burden assessed by colony forming units (CFU) $24 \mathrm{~h}$ and 2, 4 and 6 weeks post intranasal infection with $M$. avium Chester or M. avium 2-151 smt in C57BL/6 mice, $\mathrm{n}=3-7$ mice per time point, data represents mean \pm SD. (b) Lung bacterial burden assessed by CFU $24 \mathrm{~h}$ post infection by aerosol or intranasal infection, $\mathrm{n}=4$ mice per group.

C57BL/6 mice challenged with aerosol MAC 101, MAC 2285 R or MAC $2285 \mathrm{~S}^{38}$ sustain a persistent infection as measured up to 6 weeks post challenge (Supplemental Figure S1). Next, we optimized previous models using a virulent $M$. avium isolate and empirically developed the low dose aerosol (LDA) infection parameters for $M$. avium 2-151 smt (Fig. 1b), making it better aligned with the human aerosol route of infection.

In vivo growth kinetics and immune responses in C57BL/6 and Beige mice. We next evaluated the in vivo growth kinetics of M. avium 2-151 smt in both the C57BL/6 and Beige models to determine the host-induced immune response to infection, histopathology as well as potential bacterial phenotypic differences between the WT and immunocompromised hosts. Lungs and spleens were harvested and homogenates were plated $24 \mathrm{~h}, 1,2,4,6$ and 8 weeks post infection to determine bacterial burden and colony phenotypes. Not surprisingly, despite receiving the same challenge dose, Beige mice were less able to control the infection with the clinical M. avium isolate, resulting in significantly higher bacterial counts in the lung and spleen over time, from one to eight weeks post challenge (Fig. 2a,b). This accelerated increase in bacterial burden in Beige mice corresponds with a two-week delay in neutrophil and mononuclear phagocyte pulmonary influx (Fig. 2c,d) and significantly decreased percentage of pulmonary lymphocytes (Fig. 2e, Supplemental Figure S2) 6-8 weeks post infection when compared to C57BL/6 mice. Indeed, previous reports have demonstrated that a decreased neutrophil recruitment in Beige mice is in part responsible for enhanced susceptibility to intranasal challenge with M. avium when compared to C57BL/6 mice ${ }^{40,41}$. Conversely, NK cell depletion in either mouse model has historically not influenced susceptibility to M. avium infection ${ }^{40}$. We confirmed in our model of M. avium 2-151 smt aerosol challenge that the kinetics and magnitude of pulmonary NK cells was not significantly different between Beige and C57BL/6 mice out to 8 weeks post infection (Fig. 2f).

In addition to flow cytometric analysis of the kinetic influx of immune cells to the lung we also evaluated pulmonary immunopathology over the course of infection using Visiopharm software and a novel, custommade algorithm using decision forest training and classification (at Colorado State University) to detect areas of inflammation, or lesions, on hematoxylin and eosin (H\&E) stained slides from accessory lung lobes (Fig. 3a,b). The high-throughput work flow presented here alleviates the heavy time burden that was previously required ${ }^{34}$ and incorporates the entire accessory lobe in the unbiased analysis for a more progressive and reproducible scoring system. Interestingly, Beige mice are afflicted with a significantly greater proportion of lung identified as an immune lesion area as compared to C57BL/6 mice, $4-8$ weeks post infection (Fig. 3b). Indeed, accessory lobes from C57BL/ 6 mice have less than $10 \%$ of the area identified as lesion at 8 weeks post infection, whereas Beige mice reach $60 \%$ lesion area by 6 weeks post infection (Fig. 3b).

In vitro growth kinetics and compound sensitivities of rough versus smooth colony morphotypes. Upon counting CFU, colony morphotypes were categorized as smooth or rough [loss or modification of glycopeptidolipid (GPL)] in appearance from ex vivo homogenate plating. While the starting infectious material was uniformly smooth transparent (smt), in both C57BL/6 and Beige mice we observed a stochastic emergence of a minor rough morphotype population (Fig. 4a-c). Interestingly, there are conflicting reports of the impact of colony morphotype on in vivo virulence. Within MAC and M. avium some studies suggest that rough variants (lacking GPL) induce a stronger host inflammatory response ${ }^{42}$ whereas other studies observed no differences in induced host cytokine production or virulence between smooth and rough phenotypes of clinical $M$. avium isolates ${ }^{43,44}$. This includes observations that $M$. avium rough and smooth colony morphotypes do not differentially induce proinflammatory TNF- $\alpha$ from human PBMCs after $20 \mathrm{~h}$ of in vitro infection ${ }^{43}$. The impact of GPL is further complicated by NTM strain variation, as studies have demonstrated divergent mouse TNF- $\alpha$ responses induced by two smooth variants of $M$. avium ${ }^{45}$.

Infection stock of uniform smooth morphology was sub-cultured in vitro for 5 weeks where rapid emergence of rough phenotype was not observed. Indeed, rough colonies were not noted until 3 weeks of passaging 
a

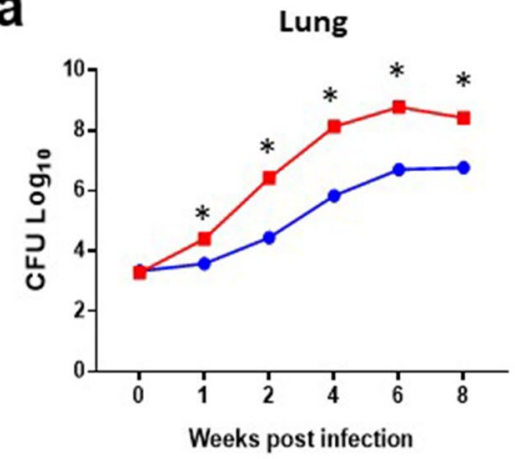

C

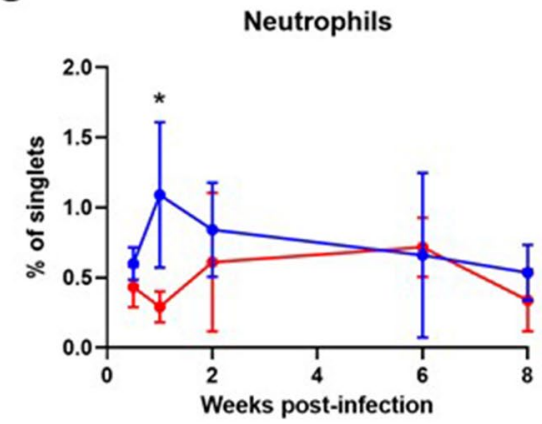

e

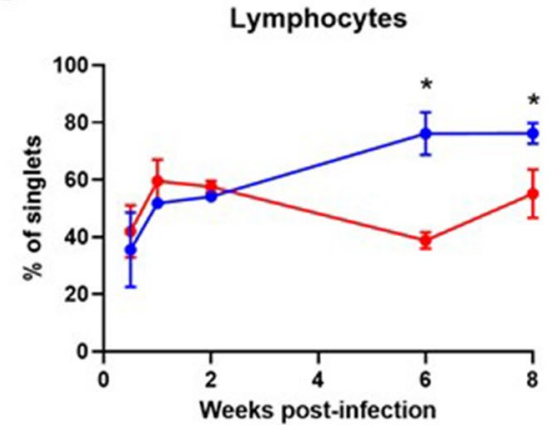

b

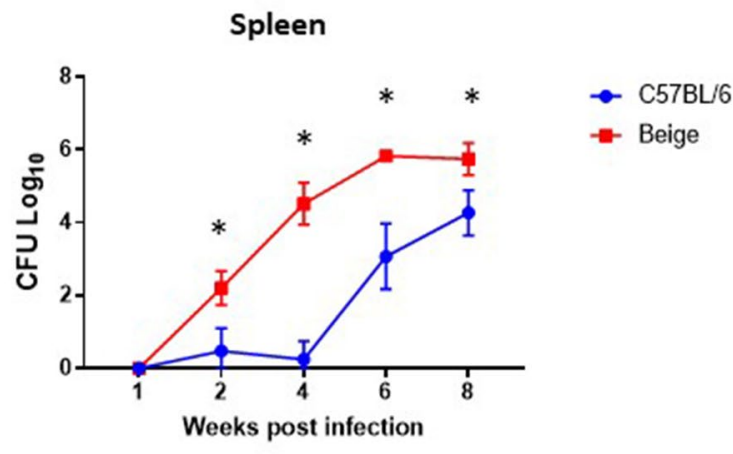

d

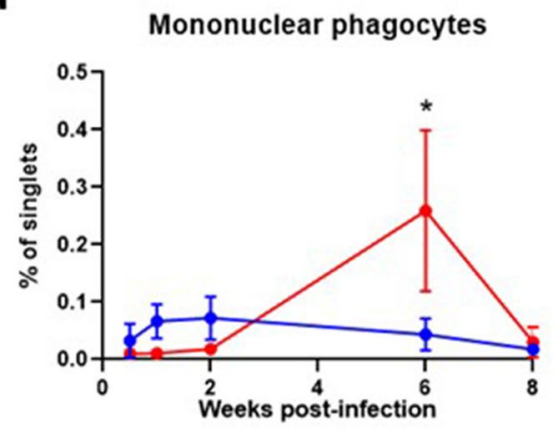

$\mathbf{f}$

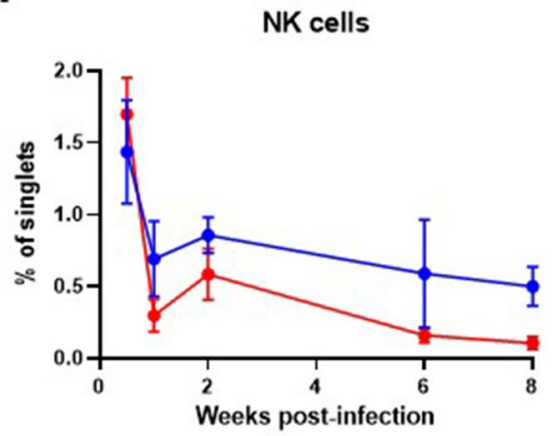

Figure 2. Beige mice sustain a greater bacterial burden of M. avium 2-151 smt over time than C57BL/6. Bacterial burden in the (a) lung and (b) spleen assessed by CFU enumeration weekly post aerosol challenge with M. avium 2-151 smt in C57BL/6 (blue circles) and Beige mice (red squares), $\mathrm{n}=4$ mice per time point. (c-f) Percent of singlet cells from lung homogenate analyzed $24 \mathrm{~h}$ to 8 weeks post infection by flow cytometry for (c) neutrophils: Ly6G + Ly6C +, (d) mononuclear phagocytes: CD64 + CD11b +, (e) Lymphocytes: Ly6G- Ly6CCD11c-CD11b- and (f) NK cells: CD64- NK1.1 +. Mean with SD represented, $n=4$ mice per time point. ${ }^{*} \mathrm{p}<0.05$, unpaired t-test with Holm-Sidak correction for multiple comparisons.

(Supplementary Table S1) in vitro whereas rough colonies were observed as early as 1 week directly ex vivo (Fig. 4).

Three rough and three smooth colonies were isolated from ex vivo lung homogenate plating and were subsequently cultured in vitro. We observed equivalent growth kinetics between smooth and rough colonies. Furthermore, when rough and smooth colonies were co-cultured in vitro the total make-up of the population retained the relative ratios of morphotypes suggesting little interference or dominance between strains in vitro (Supplementary Table S2).

There are notable differences in drug sensitivity between rough and smooth morphotypes documented for M. abscessus strains ${ }^{26,46}$, but there is little information to date for clinical M. avium isolates. Here we determined in vitro minimum inhibitory concentrations (MIC) by Optical Density (OD) following the addition of frontline antibiotics used to clinically treat MAC infections (Supplementary Table S3). We observed no significant difference in MIC between smooth and rough subcultures of M. avium 2-151 in vitro (based on a threshold of fourfold change or higher) to front line antibiotics (Supplementary Table S3). Smooth and rough variants of $M$. avium 2-151 isolated ex vivo demonstrated relatively equal sensitivities to compound inhibition and direct killing. 
a
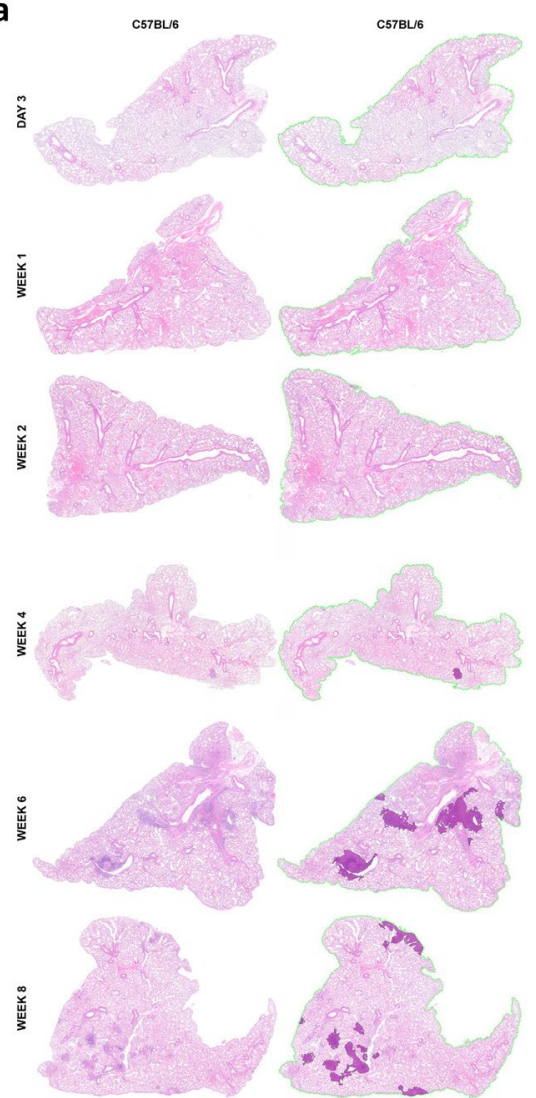
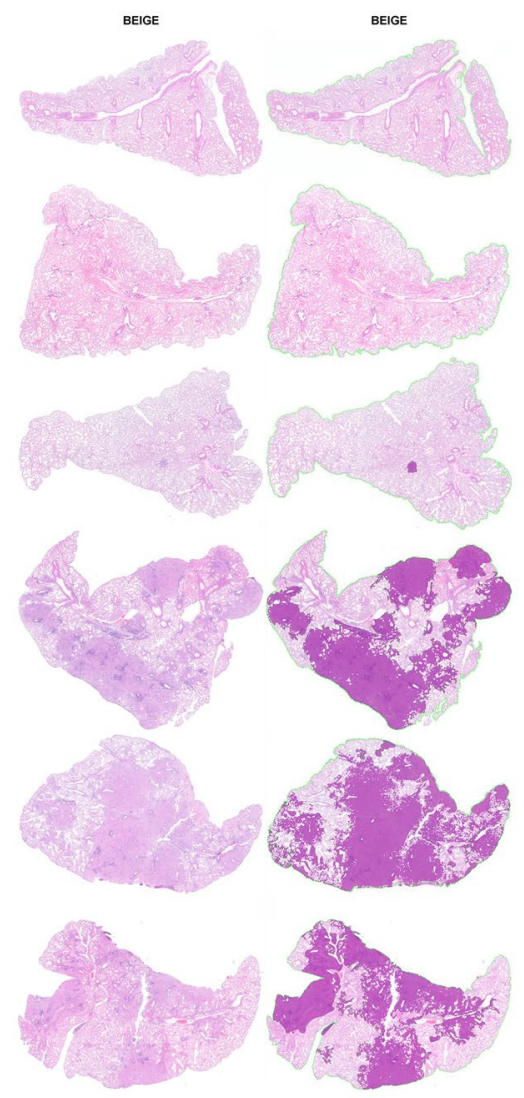

b

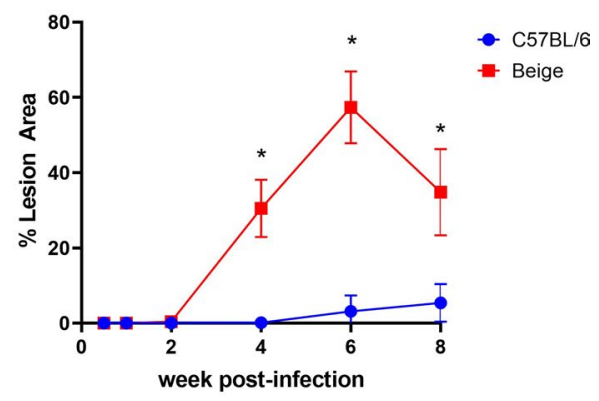

Figure 3. Kinetic quantitative histopathology using a novel high-throughput algorithm. (a) Representative accessory lung lobes from C57BL/6 (Group 1) and Beige (Group 2), before (left column) and after (right column) algorithmic scoring of $\mathrm{H} \& \mathrm{E}$ staining for pulmonary lesions (dark purple). (b) Mean $\pm \mathrm{SD}$ percent lesion area, $\mathrm{n}=3-4$ animals per group per time point. ${ }^{*} \mathrm{p}<0.05$, Two-Way ANOVA with Sidak's multiple comparison test between mouse strains at each time point.
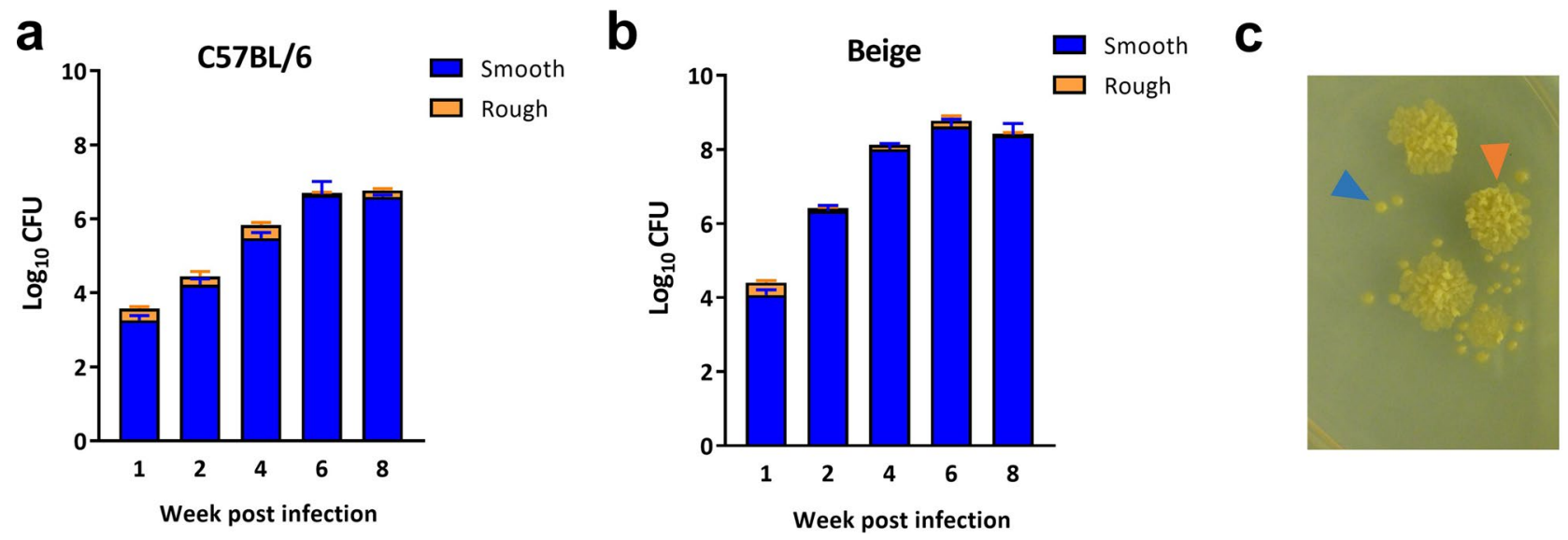

Figure 4. Stochastic emergence of rough colony morphotype in vivo. Proportion of smooth (blue) and rough (orange) morphotypes from lung homogenates 1-8 weeks post infection with M. avium 2-151 smt in (a) C57BL/6 and (b) Beige mice. (c) Representative image of rough (orange arrow) and smooth (blue arrow) colony phenotypes from an ex vivo lung homogenate agar plate culture.

Vaccine candidate vetting against NTM. Preclinical Mtb vaccine candidate, ID91, is a fusion of four Mtb proteins: Rv3619 (esxV; ESAT6-like protein), Rv2389 (RpfD), Rv3478 (PPE60) and Rv1886 (Ag85B) (Fig. 5a). These antigens were selected as candidates for Mtb vaccines based on their lack of human sequence homology and their ability to induce an ex vivo IFN- $\gamma$ response in PPD + human peripheral blood mononuclear cells (PBMC), suggesting they are immunogenic in humans ${ }^{47,48}$. Despite being derived from Mtb, these antigens share 
a

\begin{tabular}{|c|c|c|c|c|}
\hline & $\begin{array}{c}\text { Rv3619 } \\
\text { | }\end{array}$ & $\begin{array}{c}\text { Rv2389 } \\
\text { | }\end{array}$ & $\begin{array}{c}\text { Rv3478 } \\
\text { I }\end{array}$ & $\begin{array}{c}\text { Rv1886 } \\
\text { | }\end{array}$ \\
\hline ID91 & $10 \mathrm{kDa}$ & $10 \mathrm{kDa}$ & $40 \mathrm{kDa}$ & $30 \mathrm{kDa}$ \\
\hline
\end{tabular}

b

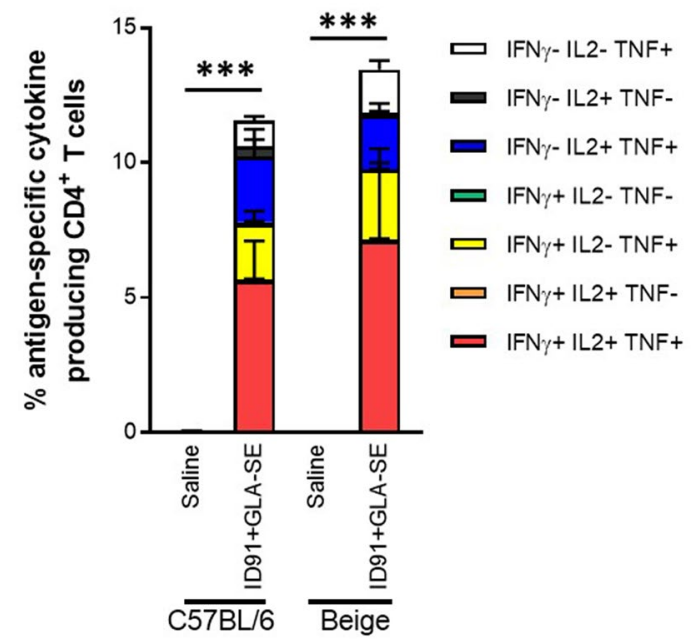

Figure 5. ID91 antigens are immunogenic in C57BL/6 and Beige mice. (a) Mtb antigens that make up the subunit fusion antigen ID91. (b) Percent of polyfunctional CD4 ${ }^{+} \mathrm{T}$ cells restimulated with ID91 antigens ex vivo from C57BL/6 or Beige mice immunized with saline or ID91 + GLA-SE, two times three weeks apart. Data represent average $\pm S D$ of $n=4$ /group, representative of two independent experiments.

\begin{tabular}{|l|l|l|l|l|}
\hline \multirow{2}{*}{ Comparison to M. avium homolog } & Mtb antigen & Rv2389 & Rv3478 & Rv1886 \\
\cline { 2 - 5 } & Rv3619 & 49 & 47 & 85 \\
\hline Percent identity & 88 & 62 & 61 & 92 \\
\hline Percent positive & 91 & $1 \mathrm{e}-36$ & $1 \mathrm{e}-57$ & 0.0 \\
\hline e-value & $4 \mathrm{e}-62$ & &
\end{tabular}

Table 1. Mycobacterial consensus of ID91 antigens. Values reported based on NCBI BLASTp of protein sequences from Mtb and M. avium antigen alignments.

significant sequence homology amongst different mycobacterial species, including M. avium (Table 1). Indeed, when we executed a theoretical epitope prediction, using the IEDB database, we observed significant overlap of predicted MCHII binding peptides in the mouse H2-iAB allelic background (Supplemental Table S4-S7) as well as predicted immunogenic $\mathrm{CD} 4^{+} \mathrm{T}$ cell epitopes across human HLAs (Supplemental Table S8). Not surprisingly, the greatest overlap in these predicted epitopes were between Rv3619 and Rv1886 antigen homologs given they have the highest sequence identity between $\mathrm{Mtb}$ and M. avium.

Therefore we leveraged this ID91-based vaccine as a proof-of-concept subunit candidate against M. avium 2-151 smt challenge in the preclinical model. Interestingly, T cell responses directed to Ag85B, an antigen in the vaccine candidate, have demonstrated some protective efficacy against $M$. avium in a P25 transgenic mouse model (the majority of TCR specific for Ag85B) akin to that observed in C57BL/6 wild type mice ${ }^{49}$. Moreover, we verified that ID91 antigens formulated in GLA-SE were immunogenic in both Beige and C57BL/6 mice. Vaccine candidate ID91 + GLA-SE was given twice intramuscularly two weeks apart and splenocytes were isolated one week after the boost immunization. Robust $\mathrm{CD}^{+} \mathrm{T}$ helper 1 responses $\left(\mathrm{T}_{\mathrm{H}} 1\right)$, including production of IFN- $\gamma$, IL-2 and TNF cytokines, were elicited from ex vivo ID91 restimulated splenocytes from ID91 + GLA-SE immunized mice but not from mice that received saline only (Fig. 5b, Supplemental Figure S3).

We next evaluated whether each mouse strain was equally sensitive and receptive to innate immune stimulation by the synthetic TLR4 agonist, GLA, by evaluating surface expression of activation and costimulatory markers on mouse bone marrow-derived dendritic cells (BMDC). As we have observed previously ${ }^{50}$, GLA induced expression of CD40 and CD86 activation markers on CD11 ${ }^{+}$cells in a dose-dependent manner (Fig. 6a,b). The percentage of CD40 and CD86 expression induced by LPS and GLA-aqueous formulation-(AF) was similar between C57BL/6 and Beige CD11 ${ }^{+}$BMDC (Fig. 6a,b). Although we detected no global deficiency in BMDC 
a

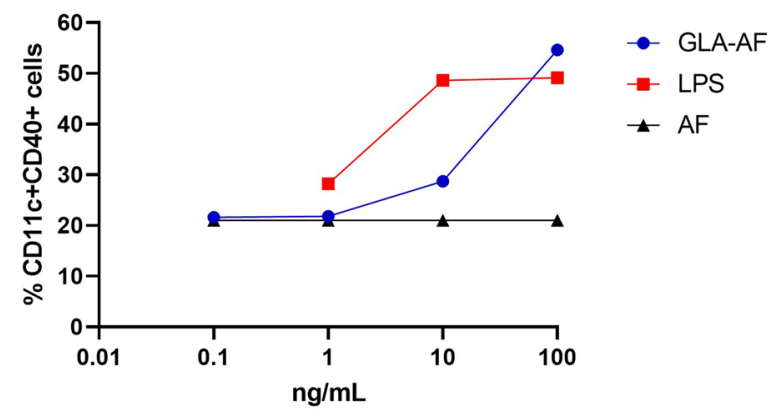

b

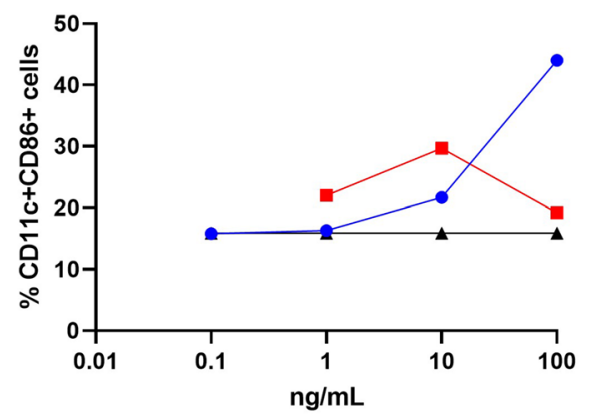

C57BL/6

C

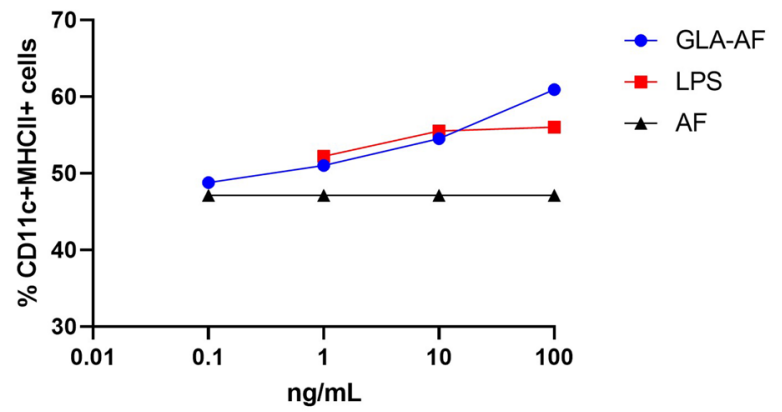

Beige

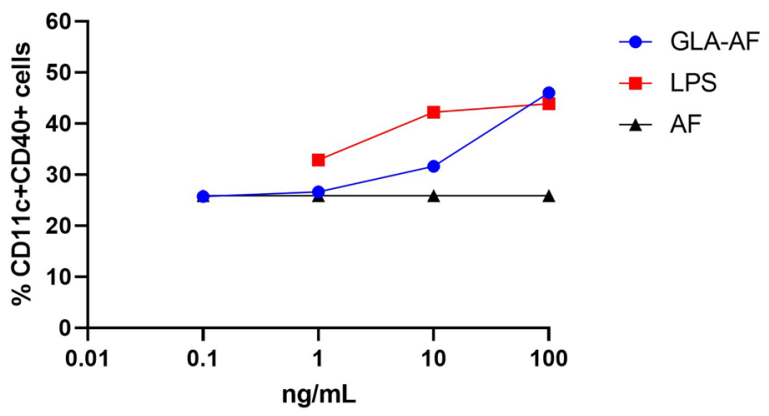

Beige

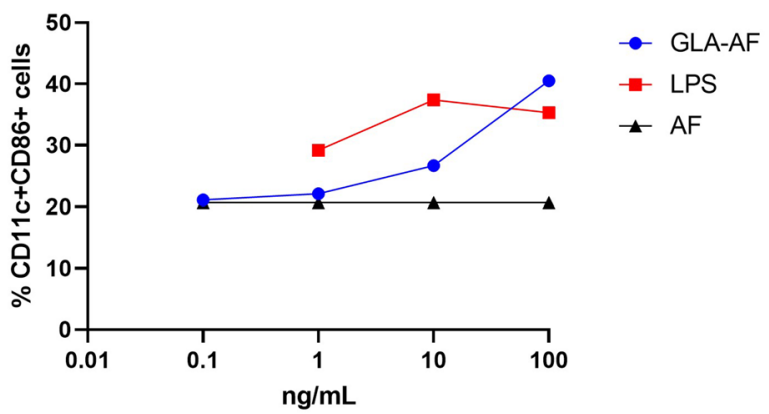

Beige

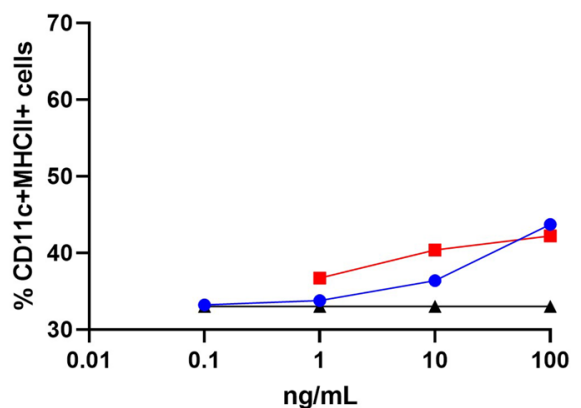

Figure 6. Activation of BMDC with GLA innate immune modulator. BMDC from C57BL/6 (left) and Beige (right) were stimulated with increasing concentrations $(0.1-100 \mathrm{ng} / \mathrm{mL})$ of GLA-AF (blue circles) and LPS (red squares) or equivalent volumes of AF (black triangles), and after $48 \mathrm{~h}$ surface stained for activation markers. CD11c positive BMDC cells were evaluated for the percentage of cells co-expressing (a) CD40, (b) CD86 and (c) MHCII.

GLA-induced activation between mouse strains we did observe dramatically lower expression of MHCII (Fig. 6c) in Beige mice across the concentrations of innate immune modulators tested compared to similar doses evaluated in $\mathrm{C} 57 \mathrm{BL} / 6$ mice.

ID91 + GLA-SE and BCG immunizations induce $T_{H} 1$ and humoral responses in both WT and immunocompromised mouse models. Bacillus Calmette-Guérin (BCG), the only licensed Mtb vaccine, has demonstrated cross-reactive immune responses against M. avium in both human PBMC and mouse lymphocytes ${ }^{51}$, and is protective against challenge with M. avium in mice ${ }^{52-54}$. Furthermore, retrospective epidemiological evidence from Finland suggests that cessation of universal BCG administration in infants across the nation has coincided with a significant increase of NTM infection and disease in children ${ }^{55}$. Route of administration of BCG is an active and promising area of research, including the exciting publication by P. A. Darrah and colleagues whereby intravenous (i.v.) and intradermal (i.d.) BCG immunizations in nonhuman primates were evaluated for efficacy against Mtb challenge ${ }^{56}$. In our mouse preclinical model, as well as those of our colleagues, BCG given i.d. has proven to be a moderately effective vaccine against $\mathrm{Mtb}^{34,57-60}$. Therefore, we utilized an i.d. administration of BCG as a positive control for our vaccine efficacy and immunogenicity evaluations.

Six weeks post infection, vaccine immunogenicity (humoral and cellular) was evaluated in cohorts of mice that were immunized with adjuvant alone (GLA-SE), ID91 + GLA-SE or BCG and challenged with a LDA of $M$. avium 2-151 smt. C57BL/6 and Beige mice immunized with ID91 + GLA-SE demonstrated robust ID91 antigenspecific (Fig. 7a-d) and Ag85B-specific (Fig. 7e-h) total IgG, IgG2c, IgG1, and IgA antibody responses, whereas GLA-SE or BCG immunized mice demonstrated much weaker ID91 and Ag85B antigen-specific humoral 
a

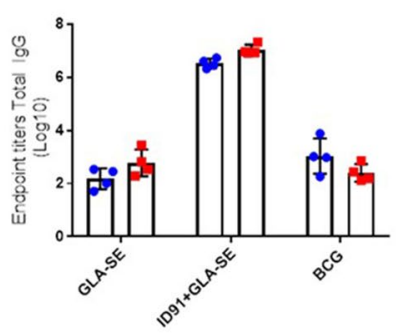

e

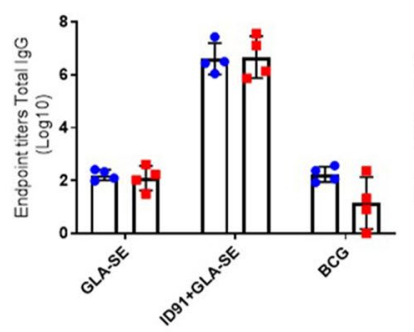

f b
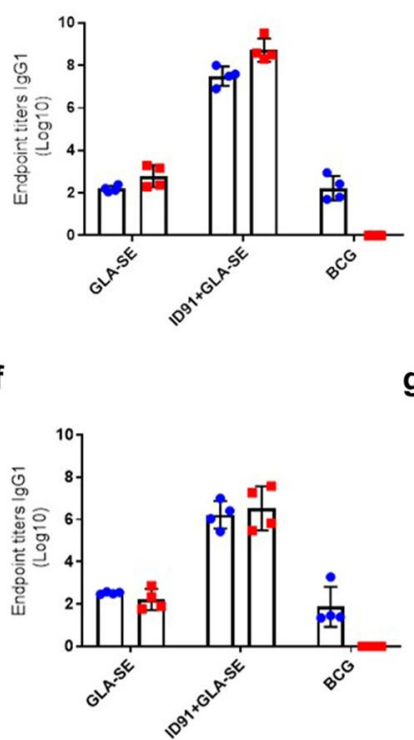

C

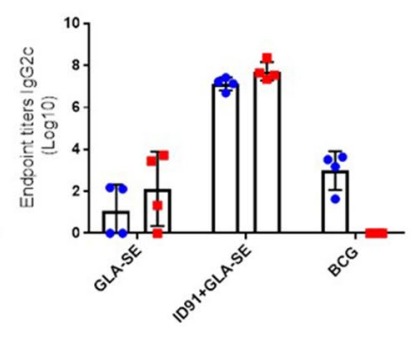

g

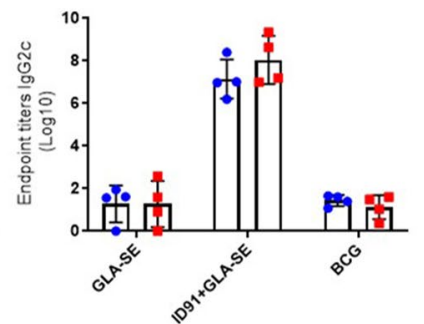

d

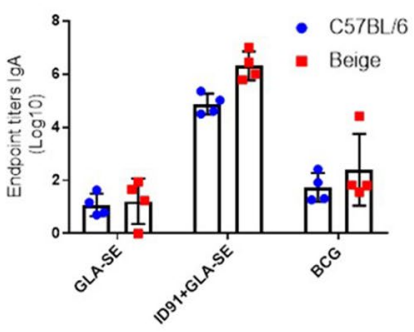

h

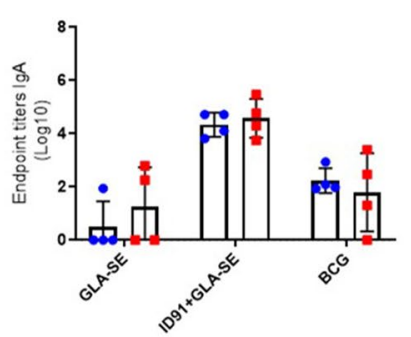

Figure 7. Humoral responses in C57BL/6 and Beige mice six weeks post-infection with M. avium 2-151 smt. Serum from prophylactically immunized C57BL/6 (blue circles) and Beige (red squares) mice with GLA-SE, ID91 + GLA-SE, or BCG was evaluated for ID91 antigen specific (a) total IgG, (b) IgG2c, (c) IgG1 and (d) IgA endpoint titers as well as Ag85B-specific (e) total IgG, (f) IgG2c, (g) IgG1 and (h) IgA endpoint titers. Bars represent average endpoint titers $\pm S D$, individual shapes represent individuals, $n=4$ /group.

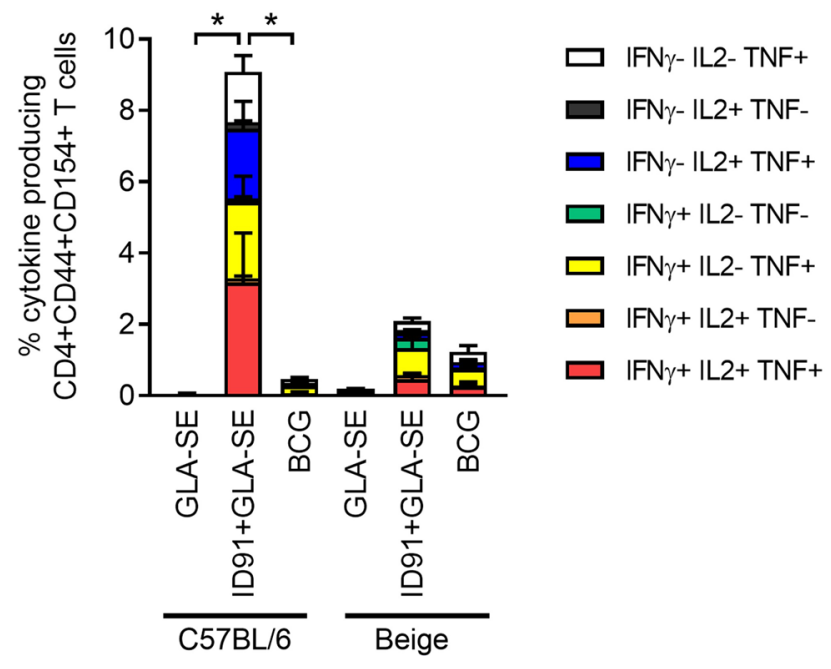

Figure 8. Greater magnitude and composition of antigen-specific polyfunctional $\mathrm{CD} 4^{+} \mathrm{T}$ cells with ID91 + GLA-SE vaccination. Pulmonary $\mathrm{T}_{\mathrm{H}} 1$ cytokine producing CD4 + CD 44 + CD154 + T cells 6 weeks post $M$. avium 2-151 smt challenge from immunized C57BL/6 or Beige mouse cohorts restimulated ex vivo with ID91. ${ }^{*} \mathrm{p}<0.05$, one-way ANOVA with Tukey's multiple comparison test.

responses. Interestingly, C57BL/6 and Beige mice seemed to induce a similar degree of each antibody subtype within each immunization cohort.

Lymphocytes were isolated from immunized cohorts six weeks post challenge from individual lung homogenates and restimulated ex vivo with ID91 antigens (Fig. 8, Supplemental Figure S3). These cells were then stained and evaluated by flow cytometry for $\mathrm{CD}^{+} \mathrm{T}_{\mathrm{H}} 1$ responses. The magnitude and composition of antigen specific polyfunctional (expressing IFN- $\gamma$, IL-2, TNF or a combination of these cytokines) CD4 ${ }^{+} \mathrm{T}_{\mathrm{H}} 1 \mathrm{~T}$ cells was significantly higher in ID91 + GLA-SE immunized C57BL/6 mice than BCG or GLA-SE immunized C57BL/6 mice (Fig. 8). Interestingly this pattern was much less robust and not significant for Beige mouse cohorts.

ID91 + GLA-SE and BCG prophylactically protect WT and immunocompromised mice from $M$. avium 2-151 smt bacterial burden. Vaccination with ID91 + GLA-SE or BCG induced significant pro- 
phylactic protection as observed by a reduction of bacterial burden (consisting of both rough and smooth morphotypes, albeit a predominately higher proportion of smooth colonies), in the lungs and spleens of C57BL/6 and Beige mice (Fig. 9a,b). Excitingly, BCG and ID91 + GLA-SE reduced lung bacterial burden in the lungs of Beige mice by 1.76 and $0.77 \log _{10} \mathrm{CFU}$, respectively, compared to adjuvant alone (Fig. 9a). Interestingly, there was no significant difference in pulmonary lesion area at 6-weeks post infection for any treatment group of C57BL/6 mice, with relatively low (<10\%) lesion scores (Fig. 9c,d). Conversely, Beige mice demonstrated significantly higher lesion area compared to C57BL/6 across all treatment arms. While no treatment was significant, prophylactic BCG immunization did trend with lower immunopathology in Beige mice (Fig. 9c,d).

A pattern of vaccine-induced protection was independently obtained from parallel studies performed by our collaborators, demonstrating significant ID91 + GLA-SE vaccine-induced protection against M. avium 2-151 smt in the C57BL/ 6 model 6 weeks post challenge (Supplementary Figure S4). These data suggest vaccination, even in an immunocompromised host setting, may be a viable approach for protection from NTM.

\section{Conclusions}

In this work, we observed a sustained infection in wild type (C57BL/6) and immunocompromised (Beige) mouse models using a virulent clinical isolate of $M$. avium. These representative mouse models will be useful for developing candidate vaccines targeting NTM in immunocompromised or immune competent individuals. Beige mice sustain a higher bacterial burden over time than C57BL/6 mice and demonstrate a differential pulmonary immune cell influx post challenge. C57BL/6 mice exhibit earlier and more robust monocyte and neutrophil pulmonary influx $24 \mathrm{~h}$ to 2 weeks post challenge, as well as an increase in lymphocytes 6 and 8 weeks post challenge compared to Beige mice, and considerably less immunopathology over time which may be directly related to their divergent ability to control infection early and late. These data represent an expansion on previous reports $s^{40,41}$, including the aerosol route of challenge and the specific concurrent quantification of lymphocytes, mononuclear phagocytes, NK cells, and neutrophils in the lungs of infected mice over time. While Beige mice do not represent a model that is defective in proinflammatory signaling axes, which can predispose humans to NTM infection, this mouse strain provides a unique opportunity as a model, where these mice can (1) sustain infection out to at least 6 weeks post challenge with NTM in spite of their slight immunodeficiencies, and can (2) still be further immunologically manipulated (e.g. cell depletion) to extrapolate vaccine-induced mechanisms of control/resistance. Interestingly, we observed a stochastic rough-colony phenotype emergence in vivo as early as 1 week following infection with M. avium 2-151smt, whereas a switch to rough morphology was less prominent in vitro suggesting that in vivo pressure(s) may be critical for this loss/modification to GPL. There was no evidence of interference between morphotypes in vitro or preferential growth kinetics. Furthermore, we did not identify differences in sensitivity to front line antibiotics in vitro between rough and smooth M. avium 2-151 morphologies.

Our proof of concept vaccine candidate ID91 + GLA-SE was readily immunogenic in both mouse models, including enhanced ID91-specific humoral and $\mathrm{CD} 4^{+} \mathrm{T}_{\mathrm{H}} 1$ cellular responses. This is an important finding given that IFN- $\gamma$ activates neutrophils and macrophages to phagocytose and/or kill intracellular pathogens, including mycobacteria such as NTM ${ }^{61-64}$. Furthermore, the positive feedback loop between IFN- $\gamma$ and IL-12 is critical for the control of mycobacteria ${ }^{65,66}$. Cellular $\mathrm{CD}^{+} \mathrm{T}_{\mathrm{H}} 1 \mathrm{~T}$ cell responses are likely a critical component for mycobacterial infection susceptibility as persons living with HIV and waning $\mathrm{CD} 4^{+} \mathrm{T}$ cell counts $(<50$ cells/ $\mu \mathrm{l})$ can present with disseminated extrapulmonary Mtb or NTM infections ${ }^{67,68}$. TNF, another prominent $\mathrm{T}_{\mathrm{H}} 1$ cytokine, has been clinically shown to help control NTM infections, which if inhibited, as with other mycobacterial infections, can result in increased risk of infection ${ }^{8,69-71}$. In cases where a genetic deficiency in the IL- $12 /$ IFN- $\gamma$ signaling axis leads to enhanced risk of NTM infection, other $\mathrm{T}_{\mathrm{H}} 1$ cytokines like TNF may be able to fill this void and reduce the overall susceptibility. The complete mechanisms of protection against NTM infection are not yet known ${ }^{17}$. Therefore, we hypothesized that a $\mathrm{T}_{\mathrm{H}} 1$ skewing vaccine candidate may afford partial protection in WT and immunocompromized preclinical models.

Indeed, both ID91 + GLA-SE and BCG demonstrate prophylactic protection against M. avium 2-151 smt aerosol challenge. Importantly, BCG immunization resulted in greater protection in both Beige and C57BL/6 mice compared to the proof of concept ID91 + GLA-SE vaccine, emphasizing the need for more work in specific antigen design and optimization for future candidates. In these experiments, pulmonary lesion scores did not align with overall bacterial burden, suggesting the mechanism of protection may well involve immune cell kinetics and be less directly related to maintaining pulmonary architecture. Although BCG was leveraged in this study as a positive control, historically live-attenuated vaccines have been contraindicated for immune compromised individuals, the same population disproportionately affected by NTM disease. BCG is not recommended for individuals living with HIV/AIDS as BCG-induced disseminated disease has been reported ${ }^{72,73}$. Furthermore, while BCG vaccination before NTM exposure induces some cross-reactive immunity ${ }^{51}$, BCG vaccination in the preclinical model after infection or exposure to NTM does not seem to reduce NTM infection or disease e $^{74}$. The complex interplay of historical mycobacterial exposures, both repeatedly to environmental NTM and BCG vaccination, is an important consideration for early vaccine design and efficacy testing. We believe that through rational vaccine platform selection and design a vaccine against NTM and specifically M. avium, can be developed as a safe strategy against this opportunistic infection.

Although outside the scope of this proof-of-concept study, it is not clear whether BCG and a subunit vaccine could provide synergistic protection against NTM. Other vaccine platform approaches, including DNA-based vaccines encoding mycobacterial antigen ${ }^{52,75}$, have not yet demonstrated synergistic protection over that of BCG alone in the preclinical model, despite inducing significant antigen-specific IFN- $\gamma^{+} \mathrm{CD}_{4}^{+} \mathrm{T}_{\mathrm{H}} 1$ responses ${ }^{52,53}$. M. avium culture filtrate protein (CFP) has also been explored as a heterologous mixed protein vaccine candidate, akin to Mtb studies ${ }^{76}$, and when formulated with DDA adjuvant was able to afford 1.0 and $0.5 \log _{10}$ CFU 
a
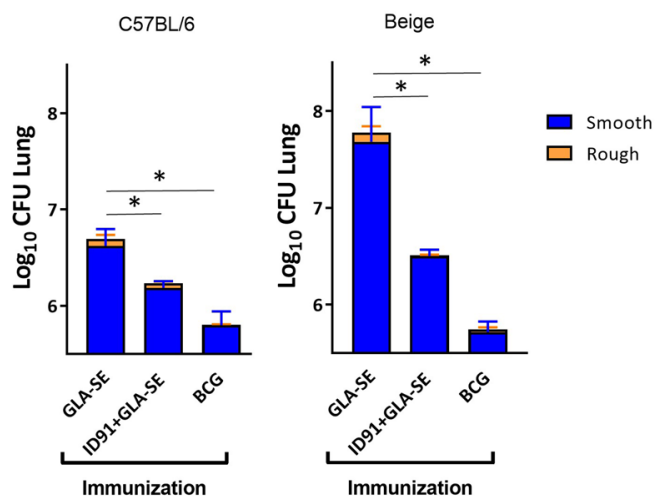

C
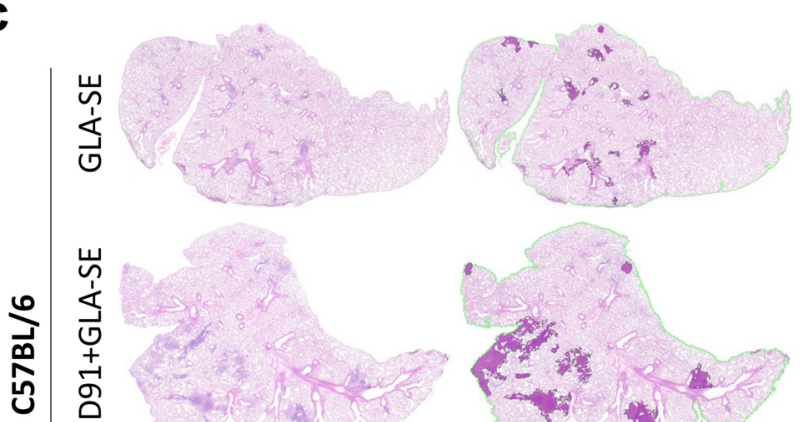

药
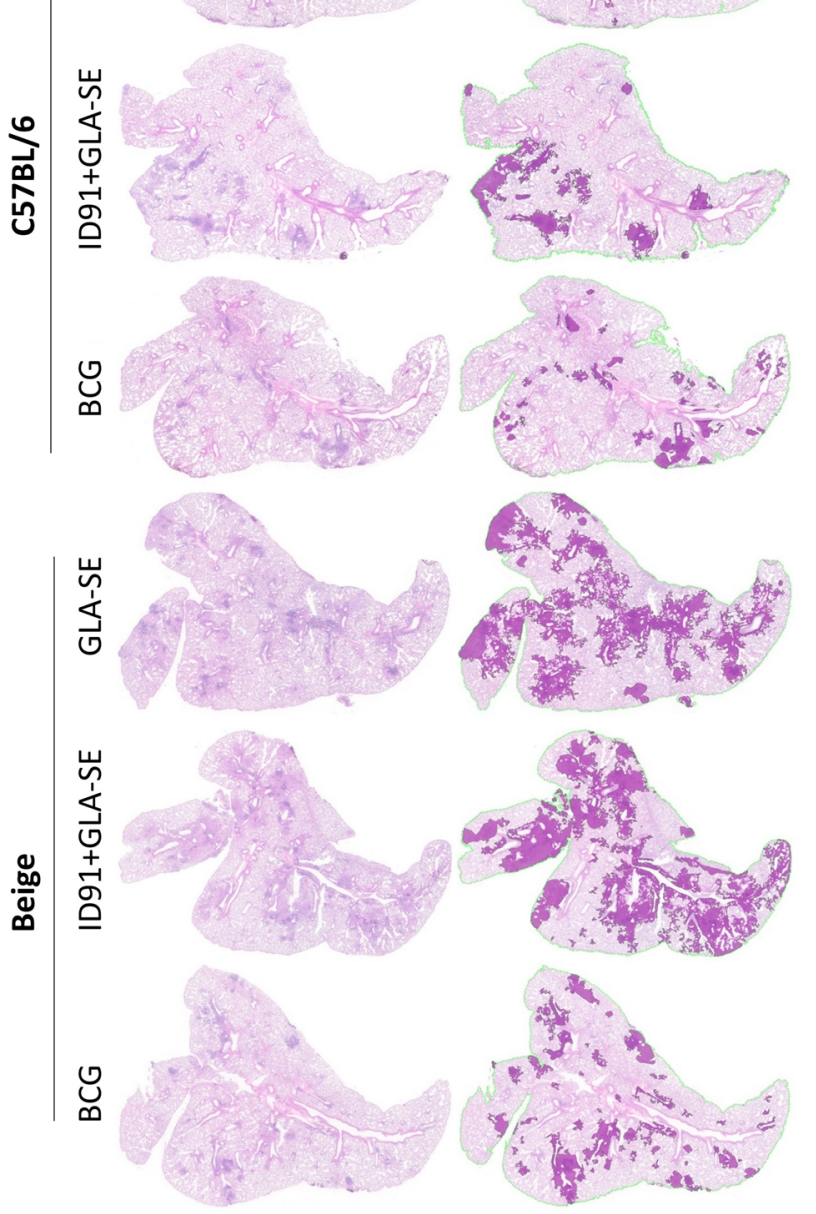

Figure 9. ID91 + GLA-SE and BCG prophylactically protect against pulmonary M. avium 2-151 smt infection. Cohorts of C57BL/6 and Beige mice were prophylactically immunized i.m. with GLA-SE, or ID91 + GLA-SE or i.d. with BCG and subsequently challenged with M. avium 2-151 smt. Bacterial burden in (a) lung and (b) spleen 6 weeks (42 days) post infection with M. avium 2-151 smt in C57BL/6 and Beige mice. Mean CFU \pm SD for $n=7$ /group. ${ }^{*} p<0.05$, significant by one-way ANOVA of total CFU. (c) Representative accessory lung lobes from C57BL/6 (top) and Beige (bottom), before (left column) and after (right column) algorithmic scoring of $\mathrm{H} \& \mathrm{E}$ staining for pulmonary lesions (dark purple). (d) Mean \pm SD percent lesion area, $\mathrm{n}=3$ /group per time point. n.s. by ANOVA with Sidak's multiple comparison test between treatments within strains. 
protection in the liver and spleen, respectively, in an intravenous $M$. avium BALB/c challenge model ${ }^{66}$. The work detailed here further demonstrates that subunit + adjuvant vaccine platforms are a viable approach for novel or complimentary interventions against $M$. avium.

As a rich collection of species with expanding prevalence and global health burden, research focus on NTM should be prioritized. Vaccine strategies that (1) develop NTM-specific antigen candidates that are immunogenic in humans and (2) devise focused regimens with respect to different disease indications (e.g. Cystic Fibrosis (CF) or anti-TNF-therapy, scenarios with defects in mechanical or physical disruptions to the pulmonary space as well as those with disrupted proinflammatory signaling), have the capacity to significantly impact the burden of NTM disease. Moreover, the successful development of a vaccine against NTM species may help inform vaccines against other mycobacteria, such as Mtb.

\section{Materials and methods}

Preclinical animal model. Female C57BL/6 mice and Beige mice 4-6 weeks of age were purchased from Charles River Laboratory and Jackson Laboratory (Sacramento, CA, USA), respectively. All methods were carried out in accordance with relevant guidelines and regulations with respect to animal welfare. Mice were housed at the Infectious Disease Research Institute (IDRI) biosafety level 3 animal facility under pathogen-free conditions and were handled in accordance with experimental protocols that were approved by IDRIs Institutional Animal Care and Use Committee (IACUC). Mice were infected either by the intranasal (i.n.) route or aerosol challenge with $10^{4} \mathrm{CFU}$ of Mycobacterium avium Chester or Mycobacterium avium 2-151 smt $\mathrm{st}^{77}$ (obtained from Dr. Diane Ordway of CSU) using a University of Wisconsin-Madison aerosol whole body exposure chamber with a Collison nebulizer. Twenty-four hours post challenge the lungs of three mice were homogenized and plated on Middlebrook 7H10 agar (Molecular Toxicology, Boone, North Carolina, USA) to confirm delivery quantity of $10^{4} \mathrm{CFU}$ per mouse. Other Female C57BL/6 mice 4-6 weeks of age were housed at Colorado State University (CSU) biosafety level 3 animal facility in accordance with the CSU IACUC. These mice were used in repeat vaccination experiments as described below or to evaluate other clinical MAC isolates for bacterial burden over time. Some of these animals were challenged via aerosol with M. avium $101^{78}$ (obtained from ATCC), M. avium $2285 \mathrm{~S}$ or M. avium $2285 \mathrm{R}^{38}$ (obtained from Dr. Kenneth N. Oliver of the NIH Pulmonary Clinical Medicine Section) using a Glas-Col whole body exposure chamber.

Bacterial burden/CFU. Bacterial counts were enumerated from 4 to 7 mice per group, 24 h to 8 weeks post infection. With methodology we have previously reported ${ }^{34}$, mice were euthanized with $\mathrm{CO}_{2}$, and lung and spleen tissue from infected animals was isolated and homogenized in $5 \mathrm{~mL}$ of either RPMI + FBS (lung) or PBS + Tween-80 (Sigma-Aldrich, St. Louis, Missouri, USA) CFU buffer (spleen) using an Omni tissue homogenizer (Omni International, Kennesaw, GA, USA) or Precellys Tissue Homogenizer (Bertin Corp. Rockville, MD, USA). Serial dilutions of homogenate were made in CFU buffer and aliquots were plated on Middlebrook $7 \mathrm{H} 10$ agar plates and subsequently incubated at $37^{\circ} \mathrm{C}$ and $5 \% \mathrm{CO}_{2}$ for $2-3$ weeks before colonies were counted. Bacterial burden, as CFU/mL, was calculated per organ and is presented here as $\log _{10}$ values. Colony morphotype (rough versus smooth appearance) was also denoted at counting. Reduction in the bacterial burden was calculated as the difference in mean $\log 10$ values between groups assessed.

Histopathology and image analysis. Lung accessory lobes were collected at different time points after M. avium 2-151 smt challenge and were immediately perfused and subsequently stored in $10 \%$ normal buffered formalin. Fixed lungs were embedded in paraffin, cut and slides were generated with hematoxylin and eosin (H\&E) staining at the Benaroya Research Institute Histology Core facility (Seattle, WA, USA), with methodology previously reported ${ }^{34}$. Stained slides were blinded and analyzed by a veterinary pathologist (Dr. Brendan Podell, Colorado State University). H\&E stained sections were scanned at 20X magnification using an Olympus VS120 microscope, Hamamatsu ORCA-R2 camera, and Olympus VS-ASW 2.9 software. Visiopharm software was used for image analysis. For each tissue section, a region of interest (ROI) was generated at a low magnification with a custom tissue detecting algorithm using decision forest training and classification to differentiate tissue versus background based on color and area. Lesions were identified within tissue ROI's at a high magnification with an additional custom-made algorithm using decision forest training and classification based on staining intensity, color normalization and deconvolution, area, and morphological features. Percent lesion calculations were integrated into the same algorithm and calculated from tissue area and lesion area as designated by the ROI and lesions detected. Lesion identification and quantification were then reviewed and edited by a pathologist as needed ${ }^{79}$.

In vitro growth kinetics. M. avium 2-151 smooth and rough colony variants were isolated from ex vivo lung homogenate plating. These isolates were grown in Middlebrook 7H9 medium supplemented with $10 \%$ v/v OADC (oleic acid, albumin, dextrose, catalase) and 0.05\% w/v Tween 80 (7H9-Tw-OADC) under aerobic conditions.

In vitro growth of $\boldsymbol{M}$. avium smooth and rough variants. M. avium 2-151 smt was inoculated at an $\mathrm{OD}_{590}$ of 0.05 into a stirring culture of 7H9-Tw-OADC. Cultures were diluted 1:10 weekly in fresh 7H9-Tw$\mathrm{OADC}$ and serial dilutions were plated onto 7H10-OADC agar plates to look for emergence of a rough variant. For competition experiments, M. avium 2-151 smooth and rough variants were inoculated at various ratios (based on $\mathrm{OD}_{590}$ ) into 7H9-Tw-OADC. Cultures were diluted 1:10 weekly in fresh 7H9-Tw-OADC and serial dilutions were plated onto 7H10-OADC agar plates. Smooth and rough colonies were counted and converted to a percentage of the overall colony count. 
Determination of minimum inhibitory concentration (MIC). MICs of selected anti-bacterial compounds were assessed with minor modifications as previously reported ${ }^{80}$. Briefly, MICs were determined against M. avium 2-151 smooth or M. avium 2-151 rough morphotypes grown in 7H9-Tw-OADC under aerobic conditions. Compounds dissolved in dimethylsulfoxide (DMSO) were added to 96 well plates using automated liquid handlers to a final DMSO concentration of $2 \%$. Bacteria was added to a starting $\mathrm{OD}_{590}$ of 0.02 and growth was measured by $\mathrm{OD}_{590}$ after 5 days of growth at $37^{\circ} \mathrm{C}$. MIC is defined as the concentration of compound required to inhibit growth of M. avium by $90 \%$ and was determined from the Levenberg-Marquardt least squares plot ${ }^{81}$.

Determination of compound killing kinetics. M. avium smt or M. avium rough variants were inoculated at $\sim 1 \times 10^{5}$ colony-forming units (CFU)/mL into 7H9-Tw OADC containing compound or DMSO alone as a control (final DMSO concentration of $2 \%$ in all samples). Standing cultures were incubated for 7 days at $37^{\circ} \mathrm{C}$, and serial dilutions were plated for CFU determination.

ID91 antigen comparison. Mtb antigens that comprise the fusion ID91 were aligned to homologs from $M$. avium using NCBI BLASTp and the compositional matrix adjust method to generate e-values, percent identity and percent positive comparisons between species of mycobacteria. The MHCII binding predictions made for each antigen were made on 12/15/2020 using the IEDB analysis resource Consensus tool ${ }^{82,83}$ (tool.iedb.org), using mouse $\mathrm{H} 2$-iAB allele selection with the consensus $(\mathrm{smm} / \mathrm{nn}$ ) method and default 15 -mer length. Low adjusted rank is considered good potential binders, and a maximum threshold of 50 was used. We also leveraged the IEDB CD4 $4^{+} \mathrm{T}$ cell Immunogenicity prediction tool ${ }^{84}$ using combined prediction methods and a maximum combined score threshold of 50 across human HLAs.

Vaccines and adjuvants. Cohorts of mice were immunized intramuscularly (i.m.) three times three weeks apart, with the final immunization occurring 4 weeks before challenge. Mice received either saline alone, or vaccinations containing $0.5 \mu \mathrm{g} / \mathrm{dose}$ of ID91 recombinant fusion protein combined with $5.0 \mu \mathrm{g} / \mathrm{dose}$ TLR4 stimulant glucopyranosyl lipid adjuvant (GLA), formulated in a squalene emulsion (SE), as previously published ${ }^{34,50,85}$. A separate cohort of mice were immunized once intradermally (i.d.) with $10^{4} \mathrm{CFU}$ of bacillus Calmette-Guérin (BCG) (Sanofi Pasteur, Theracys) 10 weeks before challenge; mice received $100 \mu \mathrm{l}$ total, $50 \mu \mathrm{l}$ on either side of the base of the tail.

Bone marrow-derived DC. BMDC were culture-induced in vitro using sterile aspirated bone marrow from the femurs of Beige and C57BL/6 mice. BMDC were prepared as previously published ${ }^{50}$. Briefly, bone marrow cell suspensions were prepared, washed and suspended at a concentration of $10^{6}$ cells $/ \mathrm{mL}$ in complete medium (RPMI 1640, 10\% fetal bovine serum, 1\% L-glutamine, 1\% penicillin/streptomycin) supplemented with $20 \mathrm{ng} / \mathrm{mL}$ recombinant mouse GM-CSF and $20 \mathrm{ng} / \mathrm{mL}$ IL-4 (PeproTech, Rocky Hill, NJ). On day 3, DC were supplemented with complete media and $20 \mathrm{ng} / \mathrm{mL}$ GM-CSF. On day 6 , non-adherent cells were plated at $2 \times 10^{5}$ cells/well in fresh complete RPMI medium, and stimulated for $48 \mathrm{~h}$ at $37^{\circ} \mathrm{C}$ with $0.1-100 \mathrm{ng} / \mathrm{mL}$ of GLA, LPS or aqueous formulation (AF) agonists as indicated in the figure legends. After $48 \mathrm{~h}$ cells were surface stained with the following antibodies: CD11c (Bv650, clone N418, Biolegend), CD86 (APC, clone GL-1, Biolegend), $\mathrm{CD} 40$ (PE/Cy7, clone 3/23, Biolegend), and MHCII I-A/I-E (eF450, clone M5/114.15.2, Invitrogen), and analyzed by flow cytometry as described below.

Antibody ELISAs. Serum was collected from mice immediately after euthanasia, six weeks post challenge. Serum was diluted 1:100 (or 1:10 for IgA) and added to plates coated with either $2 \mu \mathrm{g} / \mathrm{mL}$ of ID91 antigen or $2 \mu \mathrm{g} / \mathrm{mL}$ of Ag85B. After overnight incubation, plates were exposed to HRP-conjugated total IgG, IgG1, IgG2c, or IgA (Southern Biotech). Plates were subsequently developed with SureBlue tetramethylbenzidine substrate (KPL/Seracare) and the reaction stopped with $1 \mathrm{~N} \mathrm{H2SO}$. Plates were read at $450 \mathrm{~nm}$ with $570 \mathrm{~nm}$ background subtraction.

Flow cytometry. Intracellular flow cytometry was performed on lung homogenates $24 \mathrm{~h}$ to 8 weeks post $M$. avium infection with methodology as previously reported ${ }^{34}$. Specifically, samples were incubated in red blood cell lysis buffer (eBioscience/Thermo Fischer Scientific, Waltham, Massachusetts, USA), washed and resuspended in RPMI 1640 (Life Technologies, Carlsbad, California, USA) + 10\% fetal bovine serum (FBS) (BioWhittaker, Inc./ Lonza Radnor, Pennsylvania, USA), and subsequently aliquotted in 96-well round-bottom plates. Cells were then stimulated with media alone, $10 \mu \mathrm{g} / \mathrm{mL}$ of recombinant ID91, or $1 \mu \mathrm{g} / \mathrm{mL}$ phorbol myristate acetate (PMA) (Calbiochem) $+1 \mu \mathrm{g} / \mathrm{mL}$ ionomycin (Sigma-Aldrich, St. Louis, Missouri, USA) and incubated at $37^{\circ} \mathrm{C}$. After two hours of incubation, $1 \mu \mathrm{g} / \mu \mathrm{L}$ of GolgiPlug (BD Biosciences) was added and samples were incubated at $37^{\circ} \mathrm{C}$ for an additional $8 \mathrm{~h}$. Samples then remained at $4{ }^{\circ} \mathrm{C}$ until staining. Samples were first surface stained with fluorochrome-conjugated antibodies against mouse CD4 (Bv650, clone RM4-5, BioLegend, San Diego, California, USA), CD8 (Bv510, clone 53-6.7, BioLegend), CD44 (APC-eF780, clone IM7, Invitrogen) and $1 \mu \mathrm{g} / \mathrm{mL}$ of Fc receptor block anti-CD16/CD32 (clone 93, eBioscience) in PBS with $1 \%$ bovine serum albumin (BSA) for 10-15 min at room temperature (RT). Cells were then washed and fixed using BD Biosciences Fix/Perm reagent for 20 min at RT. Subsequently, samples were washed with BD Perm/Wash followed by intracellular staining in Perm/Wash reagent with anti-mouse IFN- $\gamma$ (PE-Cy7, clone XMG1.2, Invitrogen), IL-2 (APC, clone JES6-5H4, eBioscience), TNF- $\alpha$ (eF450, clone MP6-XT22, eBioscience), and CD154 (PerCP-710, clone MR7, eBioscience) for $15 \mathrm{~min}$ at RT. Early influx of mononuclear phagocytes, neutrophils and lymphocytes were assessed in lung homogenates 1-8 weeks post infection. Cells were surface stained with flourochrome-conjugated antibodies 
against mouse Ly6G (FITC, clone 1A8, Biolegend), Ly6C (PerCP-Cy5.5, clone HK1.4, eBioscience), MHCII I-A/I-E (eF450, clone M5/114.15.2, Invitrogen), CD11c (Bv510, clone N418, Biolegend), CD3 (Bv650, clone 17A2, Biolegend), CD19 (APC, clone 6D5, Biolegend), CD11b (Alexa700, clone M1/70, eBioscience), NK1.1 (PE, clone PK136, eBioscience), CD64 (PE-Cy7, clone X54-5/7.1, Biolegend) and $1 \mu \mathrm{g} / \mathrm{mL}$ of Fc receptor block anti-CD16/CD32 (clone 93, eBioscience) in PBS with 1\% bovine serum albumin (BSA) for 10-15 min at room temperature (RT). All antibodies were used at $10 \mu \mathrm{L} / \mathrm{mL}$. Before removing samples from the BSL3, samples were incubated in $4 \%$ paraformaldehyde for $20 \mathrm{~min}$. After wash and resuspension in PBS $+1 \%$ BSA, samples were acquired on a BD Bioscience LSRFortessa flow cytometer (BD Bioscience). Gating strategies were based on our previous publications ${ }^{34}$ and work by Yen-Rei A. Yu and colleagues ${ }^{86}$.

Statistics. Intracellular cytokines induced after $\mathrm{T}$ cell restimulation, and bacterial burden over time were compared between groups using two-way ANOVA with Sidak's multiple comparison test. Bacterial burden at a single time point was assessed using one-way ANOVA with Sidak's multiple comparison test compared to groups immunized with adjuvant alone. Flow cytometry data was assessed using FlowJo v10 (BD) and SPICE (NIH) using the Wilcoxon signed rank test. Statistical analyses, aside from flow cytometry, were performed using GraphPad Prism 7 software. p values $<0.05$ were considered significant and labeled accordingly in the figures, with methodology our group has previously reported ${ }^{34}$.

\section{Data availability}

The datasets generated and/or analyzed during the study presented here are available from the corresponding authors on reasonable request.

Received: 21 April 2020; Accepted: 5 April 2021

Published online: 27 April 2021

\section{References}

1. Strollo, S. E., Adjemian, J., Adjemian, M. K. \& Prevots, D. R. The burden of pulmonary nontuberculous mycobacterial disease in the United States. Ann. Am. Thorac. Soc. 12, 1458-1464. https://doi.org/10.1513/AnnalsATS.201503-173OC (2015).

2. Khan, K., Wang, J. \& Marras, T. K. Nontuberculous mycobacterial sensitization in the United States: National trends over three decades. Am. J. Respir. Crit. Care Med. 176, 306-313. https://doi.org/10.1164/rccm.200702-201OC (2007).

3. Vinnard, C. et al. Deaths related to nontuberculous mycobacterial infections in the United States, 1999-2014. Ann. Am. Thorac. Soc. 13, 1951-1955. https://doi.org/10.1513/AnnalsATS.201606-474BC (2016).

4. Ratnatunga, C. N. et al. The rise of non-tuberculosis mycobacterial lung disease. Front. Immunol. 11, 303. https://doi.org/10.3389/ fimmu.2020.00303 (2020).

5. Brode, S. K., Daley, C. L. \& Marras, T. K. The epidemiologic relationship between tuberculosis and non-tuberculous mycobacterial disease: A systematic review. Int. J. Tuberc Lung Dis. 18, 1370-1377. https://doi.org/10.5588/ijtld.14.0120 (2014).

6. Honda, J. R., Knight, V. \& Chan, E. D. Pathogenesis and risk factors for nontuberculous mycobacterial lung disease. Clin. Chest Med. 36, 1-11. https://doi.org/10.1016/j.ccm.2014.10.001 (2015).

7. Andréjak, C. et al. Chronic respiratory disease, inhaled corticosteroids and risk of non-tuberculous mycobacteriosis. Thorax 68, 256-262. https://doi.org/10.1136/thoraxjnl-2012-201772 (2013).

8. Winthrop, K. et al. Mycobacterial diseases and antitumour necrosis factor therapy in USA. Ann. Rheum. Dis. 72, 37-42. https:// doi.org/10.1136/annrheumdis-2011-200690 (2013).

9. Dirac, M. A. et al. Environment or host?: A case-control study of risk factors for Mycobacterium avium complex lung disease. Am. J. Respir. Crit. Care Med. 186, 684-691. https://doi.org/10.1164/rccm.201205-0825OC (2012).

10. Falkinham, J. O. Environmental sources of nontuberculous mycobacteria. Clin. Chest Med. 36, 35-41. https://doi.org/10.1016/j. ccm.2014.10.003 (2015).

11. Brode, S. K., Daley, C. L. \& Marras, T. K. The epidemiologic relationship between tuberculosis and non-tuberculous mycobacterial disease: a systematic review. Int. J. Tuberc. Lung Dis. 18, 1370-1377. https://doi.org/10.5588/ijtld.14.0120 (2014).

12. Orme, I. M. \& Ordway, D. J. Host response to nontuberculous mycobacterial infections of current clinical importance. Infect. Immun. 82, 3516-3522. https://doi.org/10.1128/IAI.01606-13 (2014).

13. Spaulding, A. B. et al. Geographic distribution of nontuberculous mycobacterial species identified among clinical isolates in the United States, 2009-2013. Ann. Am. Thorac. Soc. 14, 1655-1661. https://doi.org/10.1513/AnnalsATS.201611-860OC (2017).

14. Dartois, V., Sizemore, C. \& Dick, T. Editorial: NTM—the new uber-bugs. Front. Microbiol. 10. https://doi.org/10.3389/fmicb.2019. 01299 (2019).

15. Gopinath, K. \& Singh, S. Non-tuberculous mycobacteria in TB-endemic countries: Are we neglecting the danger?. PLoS Negl. Trop. Dis. 4, e615. https://doi.org/10.1371/journal.pntd.0000615 (2010).

16. Larsson, L.-O. et al. Pulmonary disease by non-tuberculous mycobacteria: clinical management, unmet needs and future perspectives. Expert Rev. Respir. Med. 11, 977-989. https://doi.org/10.1080/17476348.2017.1386563 (2017).

17. Baldwin, S. L., Larsen, S. E., Ordway, D., Cassell, G. \& Coler, R. N. The complexities and challenges of preventing and treating nontuberculous mycobacterial diseases. PLoS Negl. Trop. Dis. 13, e0007083-e0007083. https://doi.org/10.1371/journal.pntd.00070 83 (2019).

18. Maiga, M. et al. Failure to recognize nontuberculous mycobacteria leads to misdiagnosis of chronic pulmonary tuberculosis. PLoS ONE 7, e36902-e36902. https://doi.org/10.1371/journal.pone.0036902 (2012).

19. Jones, M. M. et al. Epidemiology of nontuberculous mycobacterial infections in the U.S. Veterans Health Administration. PLOS ONE 13, e0197976. https://doi.org/10.1371/journal.pone.0197976 (2018).

20. Ryu, Y. J., Koh, W.-J. \& Daley, C. L. Diagnosis and treatment of nontuberculous mycobacterial lung disease: clinicians' perspectives. Tuberculosis Respir. Dis. 79, 74-84. https://doi.org/10.4046/trd.2016.79.2.74 (2016).

21. Griffith, D. E. et al. An official ATS/IDSA statement: diagnosis, treatment, and prevention of nontuberculous mycobacterial diseases. Am. J. Respir. Crit. Care Med. 175, 367-416. https://doi.org/10.1164/rccm.200604-571ST (2007).

22. Muanda, F. T., Sheehy, O. \& Bérard, A. Use of antibiotics during pregnancy and risk of spontaneous abortion. Can. Med. Assoc. J. 189, E625-E633. https://doi.org/10.1503/cmaj.161020 (2017).

23. Kwon, Y. S., Koh, W. J. \& Daley, C. L. Treatment of Mycobacterium avium complex pulmonary disease. Tuberculosis Respir. Dis. 82, 15-26. https://doi.org/10.4046/trd.2018.0060 (2019).

24. Falkinham, J. O. 3rd. Epidemiology of infection by nontuberculous mycobacteria. Clin. Microbiol. Rev. 9, 177-215 (1996).

25. Koh, W.-J. et al. Treatment of refractory Mycobacterium avium complex lung disease with a moxifloxacin-containing regimen. Antimicrob. Agents Chemother. 57, 2281-2285. https://doi.org/10.1128/aac.02281-12 (2013). 
26. van Ingen, J., Boeree, M. J., van Soolingen, D. \& Mouton, J. W. Resistance mechanisms and drug susceptibility testing of nontuberculous mycobacteria. Drug Resist. Updates 15, 149-161. https://doi.org/10.1016/j.drup.2012.04.001 (2012).

27. Jhun, B. W. et al. Amikacin inhalation as salvage therapy for refractory nontuberculous mycobacterial lung disease. Antimicrob. Agents Chemother. 62, e00011-00018. https://doi.org/10.1128/aac.00011-18 (2018).

28. Daniel-Wayman, S. et al. Advancing translational science for pulmonary NTM infections: A roadmap for research. Am. J. Respir. Crit. Care Med. https://doi.org/10.1164/rccm.201807-1273PP.

29. Saunders, B. M. \& Cheers, C. Intranasal infection of beige mice with Mycobacterium avium complex: Role of neutrophils and natural killer cells. Infect. Immun. 64, 4236-4241. https://doi.org/10.1128/iai.64.10.4236-4241.1996 (1996).

30. Amaral, E. P. et al. Difference in virulence of Mycobacterium avium isolates sharing indistinguishable DNA fingerprint determined in murine model of lung infection. PLoS ONE 6, e21673. https://doi.org/10.1371/journal.pone.0021673 (2011).

31. Haug, M. et al. Dynamics of immune effector mechanisms during infection with Mycobacterium avium in C57BL/6 mice. Immunology 140, 232-243. https://doi.org/10.1111/imm.12131 (2013).

32. Rampacci, E., Stefanetti, V., Passamonti, F. \& Henao-Tamayo, M. Preclinical models of nontuberculous mycobacteria infection for early drug discovery and vaccine research. Pathogens 9,1 . https://doi.org/10.3390/pathogens 9080641 (2020).

33. Cha, S. B. et al. Experimental reactivation of pulmonary Mycobacterium avium complex infection in a modified cornell-like murine model. PLoS ONE 10, e0139251. https://doi.org/10.1371/journal.pone.0139251 (2015).

34. Larsen, S. E. et al. Enhanced Anti-Mycobacterium tuberculosis Immunity over Time with Combined Drug and Immunotherapy Treatment. Vaccines (Basel) 6. https://doi.org/10.3390/vaccines6020030 (2018).

35. Bannai, M. et al. Disparate effect of beige mutation on cytotoxic function between natural killer and natural killer $\mathrm{T}$ cells. Immunology 100, 165-169. https://doi.org/10.1046/j.1365-2567.2000.00040.x (2000).

36. Roder, J. C., Lohmann-Matthes, M.-L., Domzig, W. \& Wigzell, H. The Beige Mutation in the Mouse. II. Selectivity of the Natural Killer (NK) Cell Defect 123, 2174-2181 (1979).

37. Pflumio, F., Fonteneau, P. \& Loor, F. Impaired antibody response of C57BL/ 6 beige mutant mice to a thymus-independent type 2 antigen. Immunol. Lett. 23, 269-274. https://doi.org/10.1016/0165-2478(90)90071-W (1990).

38. Verma, D. et al. Mycobacterium avium infection in a C3HeB/FeJ mouse model. Front. Microbiol. 10, 693-693. https://doi.org/10. 3389/fmicb.2019.00693 (2019).

39. Lounis, N. et al. Impacts of interleukin-12 on multiplication of Mycobacterium tuberculosis and Mycobacterium avium complex in mice. Clin. Microbiol. Infect. 5, 331-338. https://doi.org/10.1111/j.1469-0691.1999.tb00152.x (1999).

40. Florido, M., Appelberg, R., Orme, I. M. \& Cooper, A. M. Evidence for a reduced chemokine response in the lungs of beige mice infected with Mycobacterium avium. Immunology 90, 600-606. https://doi.org/10.1046/j.1365-2567.1997.00206.x (1997).

41. Appelberg, R., Castro, A. G., Gomes, S., Pedrosa, J. \& Silva, M. T. Susceptibility of beige mice to Mycobacterium avium: Role of neutrophils. Infect. Immun. 63, 3381-3387 (1995).

42. Bhatnagar, S. \& Schorey, J. S. Elevated mitogen-activated protein kinase signalling and increased macrophage activation in cells infected with a glycopeptidolipid-deficient Mycobacterium avium. Cell. Microbiol. 8, 85-96. https://doi.org/10.1111/j.1462-5822. 2005.00602.x (2006).

43. Sampaio, E. P. et al. Mycobacterium abscessus and M. avium trigger Toll-like receptor 2 and distinct cytokine response in human cells. Am. J. Respir. Cell Mol. Biol. 39, 431-439. https://doi.org/10.1165/rcmb.2007-0413OC (2008).

44. Pedrosa, J. et al. Characterization of the virulence of Mycobacterium avium complex (MAC) isolates in mice. Clin. Exp. Immunol. 98, 210-216 (1994).

45. Furney, S. K., Skinner, P. S., Roberts, A. D., Appelberg, R. \& Orme, I. M. Capacity of Mycobacterium avium isolates to grow well or poorly in murine macrophages resides in their ability to induce secretion of tumor necrosis factor. Infect. Immun. 60, 4410-4413 (1992).

46. Greendyke, R. \& Byrd, T. F. Differential antibiotic susceptibility of Mycobacterium abscessus variants in biofilms and macrophages compared to that of planktonic bacteria. Antimicrob. Agents Chemother. 52, 2019-2026. https://doi.org/10.1128/AAC.00986-07 (2008).

47. Orr, M. T. et al. Immune subdominant antigens as vaccine candidates against Mycobacterium tuberculosis. J. Immunol. (Baltimore, Md. : 1950) 193, 2911-2918. https://doi.org/10.4049/jimmunol.1401103 (2014).

48. Bertholet, S. et al. Identification of human T cell antigens for the development of vaccines against Mycobacterium tuberculosis. J. Immunol. (Baltimore, Md. : 1950) 181, 7948-7957 (2008).

49. Cerqueira-Rodrigues, B., Mendes, A., Correia-Neves, M. \& Nobrega, C. Ag85-focused T-cell immune response controls Mycobacterium avium chronic infection. PLoS ONE 13, e0193596-e0193596. https://doi.org/10.1371/journal.pone.0193596 (2018).

50. Coler, R. N. et al. Development and characterization of synthetic glucopyranosyl lipid adjuvant system as a vaccine adjuvant. PLoS ONE 6, e16333. https://doi.org/10.1371/journal.pone.0016333 (2011).

51. Abate, G., Hamzabegovic, F., Eickhoff, C. S. \& Hoft, D. F. BCG Vaccination induces M. avium and M. abscessus cross-protective immunity. Front. Immunol. 10. https://doi.org/10.3389/fimmu.2019.00234 (2019).

52. Martin, E., Kamath, A. T., Triccas, J. A. \& Britton, W. J. Protection against virulent Mycobacterium avium infection following DNA vaccination with the 35-kilodalton antigen is accompanied by induction of gamma interferon-secreting CD4(+) T cells. Infect. Immun. 68, 3090-3096. https://doi.org/10.1128/iai.68.6.3090-3096.2000 (2000).

53. Martin, E., Triccas, J. A., Kamath, A. T., Winter, N. \& Britton, W. J. Comparative protective effects of recombinant DNA and Mycobacterium bovis bacille Calmette-Guérin vaccines against M. avium infection. Clin. Exp. Immunol. 126, 482-487. https://doi.org/ 10.1046/j.1365-2249.2001.01708.x (2001).

54. Orme, I. M. \& Collins, F. M. Prophylactic effect in mice of BCG vaccination against non-tuberculous mycobacterial infections. Tubercle 66, 117-120. https://doi.org/10.1016/0041-3879(85)90076-5 (1985).

55. Kontturi, A., Soini, H., Ollgren, J. \& Salo, E. Increase in childhood nontuberculous mycobacterial infections after bacille calmetteguérin coverage drop: A nationwide, population-based retrospective study, Finland, 1995-2016. Clin. Infect. Dis. 67, 1256-1261. https://doi.org/10.1093/cid/ciy241 (2018).

56. Darrah, P. A. et al. Prevention of tuberculosis in macaques after intravenous BCG immunization. Nature 577, 95-102. https://doi. org/10.1038/s41586-019-1817-8 (2020).

57. Baldwin, S. L. et al. Protection and long-lived immunity induced by the ID93/GLA-SE vaccine candidate against a clinical Mycobacterium tuberculosis Isolate. Clin. Vaccine Immunol. 23, 137-147. https://doi.org/10.1128/CVI.00458-15 (2016).

58. Bertholet, S. et al. A defined tuberculosis vaccine candidate boosts BCG and protects against multidrug-resistant Mycobacterium tuberculosis. Sci. Transl. Med. 2, 53ra74. https://doi.org/10.1126/scitranslmed.3001094 (2010).

59. Coler, R. N. et al. Therapeutic immunization against Mycobacterium tuberculosis is an effective adjunct to antibiotic treatment. J. Infect. Dis. 207, 1242-1252. https://doi.org/10.1093/infdis/jis425 (2013).

60. Kwon, K. W. et al. Long-term protective efficacy with a BCG-prime ID93/GLA-SE boost regimen against the hyper-virulent Mycobacterium tuberculosis strain K in a mouse model. Sci. Rep. 9, 15560. https://doi.org/10.1038/s41598-019-52146-0 (2019).

61. Tau, G. \& Rothman, P. Biologic functions of the IFN-gamma receptors. Allergy 54, 1233-1251 (1999).

62. Lai, H.-C. et al. NK Cell-derived IFN- $\gamma$ protects against nontuberculous mycobacterial lung infection. J. Immunol. ji1800123. https://doi.org/10.4049/jimmunol.1800123 (2018).

63. Hartmann, P. et al. Phagocytosis and killing of Mycobacterium avium complex by human neutrophils. J. Leukoc. Biol. 69, 397-404. https://doi.org/10.1189/jlb.69.3.397 (2001). 
64. Kedzierska, K. et al. Interferon-gamma therapy activates human monocytes for enhanced phagocytosis of Mycobacterium avium complex in HIV-infected individuals. HIV Clin. Trials 5, 80-85. https://doi.org/10.1310/T4R0-WBPL-Q2Q6-TU49 (2004).

65. Chan, E. D., Bai, X., Kartalija, M., Orme, I. M. \& Ordway, D. J. Host Immune Response to Rapidly Growing Mycobacteria, an Emerging Cause of Chronic Lung Disease. Am. J. Respir. Cell Mol. Biol. 43, 387-393. https://doi.org/10.1165/rcmb.2009-0276TR (2010).

66. Silva, R. A., Pais, T. F. \& Appelberg, R. Evaluation of IL-12 in immunotherapy and vaccine design in experimental Mycobacterium avium Infections. J. Immunol. 161, 5578-5585 (1998).

67. Society, A. T. Mycobacterioses and the acquired immunodeficiency syndrome. Am. Rev. Respir. Dis. 136, 492-496. https://doi.org/ 10.1164/ajrccm/136.2.492 (1987).

68. Holland, S. M. Nontuberculous mycobacteria. Am. J. Med. Sci. 321, 49-55. https://doi.org/10.1097/00000441-200101000-00008 (2001).

69. Winthrop, K. L., Chang, E., Yamashita, S., Iademarco, M. F. \& LoBue, P. A. Nontuberculous mycobacteria infections and anti-tumor necrosis factor-alpha therapy. Emerg. Infect. Dis. 15, 1556-1561. https://doi.org/10.3201/eid1510.090310 (2009).

70. Yoo, J.-W. et al. Mycobacterial diseases developed during anti-tumour necrosis factor-a therapy. Eur. Respir. J. 44, 1289-1295. https://doi.org/10.1183/09031936.00063514 (2014).

71. Brode, S. K. et al. Increased risk of mycobacterial infections associated with anti-rheumatic medications. Thorax 70, 677-682. https://doi.org/10.1136/thoraxjnl-2014-206470 (2015).

72. Boudes, P., Sobel, A., Deforges, L. \& Leblic, E. Disseminated Mycobacterium bovis infection from BCG vaccination and HIV infection. JAMA 262, 2386-2386. https://doi.org/10.1001/jama.1989.03430170046020 (1989).

73. Hesseling, A. C. et al. Danish bacille calmette-guerin vaccine-induced disease in human immunodeficiency virus-infected children. Clin. Infect. Dis. 37, 1226-1233 (2003).

74. Orme, I. M., Roberts, A. R. \& Collins, F. M. Lack of evidence for a reduction in the efficacy of subcutaneous BCG vaccination in mice infected with nontuberculous mycobacteria. Tubercle 67, 41-46. https://doi.org/10.1016/0041-3879(86)90030-9 (1986).

75. Velaz-Faircloth, M., Cobb, A. J., Horstman, A. L., Henry, S. C. \& Frothingham, R. Protection against Mycobacterium avium by DNA vaccines expressing mycobacterial antigens as fusion proteins with green fluorescent protein. Infect. Immun. 67, 4243-4250 (1999).

76. Orme, I. M., Andersen, P. \& Boom, W. H. T cell response to Mycobacterium tuberculosis. J. Infect. Dis. 167, 1481-1497. https://doi. org/10.1093/infdis/167.6.1481 (1993).

77. Appelberg, R. \& Orme, I. M. Effector mechanisms involved in cytokine-mediated bacteriostasis of Mycobacterium avium infections in murine macrophages. Immunology 80, 352-359 (1993).

78. Bermudez, L. E., Nash, K., Petrofsky, M., Young, L. S. \& Inderlied, C. B. Clarithromycin-resistant mycobacterium avium is still susceptible to treatment with clarithromycin and is virulent in mice. Antimicrob. Agents Chemother. 44, 2619-2622. https://doi. org/10.1128/aac.44.10.2619-2622.2000 (2000).

79. Asay, B. C. et al. Digital image analysis of heterogeneous tuberculosis pulmonary pathology in non-clinical animal models using deep convolutional neural networks. Sci. Rep. 10, 6047. https://doi.org/10.1038/s41598-020-62960-6 (2020).

80. Ollinger, J. et al. A dual read-out assay to evaluate the potency of compounds active against Mycobacterium tuberculosis. PLoS ONE 8, e60531-e60531. https://doi.org/10.1371/journal.pone.0060531 (2013).

81. Lambert, R. J. W. \& Pearson, J. Susceptibility testing: accurate and reproducible minimum inhibitory concentration (MIC) and non-inhibitory concentration (NIC) values. J. Appl. Microbiol. 88, 784-790. https://doi.org/10.1046/j.1365-2672.2000.01017.x (2000).

82. Wang, P. et al. A systematic assessment of MHC class II peptide binding predictions and evaluation of a consensus approach. PLoS Comput. Biol 4, e1000048. https://doi.org/10.1371/journal.pcbi.1000048 (2008).

83. Wang, P. et al. Peptide binding predictions for HLA DR, DP and DQ molecules. BMC Bioinf. 11, 568. https://doi.org/10.1186/ 1471-2105-11-568 (2010).

84. Dhanda, S. K. et al. Predicting HLA CD4 Immunogenicity in Human Populations. Front. Immunol. 9, 1. https://doi.org/10.3389/ fimmu.2018.01369 (2018).

85. Bertholet, S. et al. A defined tuberculosis vaccine candidate boosts BCG and protects against multidrug-resistant Mycobacterium tuberculosis. Sci. Transl. Med. 2, 53. https://doi.org/10.1126/scitranslmed.3001094 (2010).

86. Yu, Y.-R.A. et al. A protocol for the comprehensive flow cytometric analysis of immune cells in normal and inflamed murine nonlymphoid tissues. PLoS ONE 11, e0150606-e0150606. https://doi.org/10.1371/journal.pone.0150606 (2016).

\section{Acknowledgements}

The authors would like to express their gratitude to Seattle Children's Research Institute Leadership for their guidance, compassion and mentorship. The authors would also like to thank Dr. Tanya Parish (SCRI) for helpful discussions about this work. Lastly, the authors would like to thank Dr. Anne J. Lenaerts (CSU) who was co-PI on funding related to data generated in this manuscript.

\section{Author contributions}

S.E.L., R.N.C., S.L.B. and D.O. designed experiments; S.E.L., V.A.R., T.P., B.J.B., S.K.C., G.B., B.K.P., M.H.T. carried out experiments and assisted with analysis; S.E.L. wrote the manuscript and prepared figures with assistance from B.J.B., S.L.B., D.O. and R.N.C. All authors reviewed the manuscript.

\section{Funding}

Research reported here was supported by National Institute of Allergy and Infectious Diseases (NIAID) of the National Institutes of Health under award numbers R01AI125160, R21AI142267-01A1, R21AIO99534-02 and AIO99534-02, and NIAID task order HHSN272201000009I/HHSN27200001, subtask 6. SEL was supported through the University of Washington, Diseases of Public Health Importance T32 training grant number A1075090. The content is solely the responsibility of the authors and does not necessarily represent the official views of the National Institutes of Health.

\section{Competing interests}

The authors declare no competing interests.

\section{Additional information}

Supplementary Information The online version contains supplementary material available at https:/doi.org/ 10.1038/s41598-021-88291-8. 
Correspondence and requests for materials should be addressed to S.L.B. or R.N.C.

Reprints and permissions information is available at www.nature.com/reprints.

Publisher's note Springer Nature remains neutral with regard to jurisdictional claims in published maps and institutional affiliations.

(c) (i) Open Access This article is licensed under a Creative Commons Attribution 4.0 International cc) License, which permits use, sharing, adaptation, distribution and reproduction in any medium or format, as long as you give appropriate credit to the original author(s) and the source, provide a link to the Creative Commons licence, and indicate if changes were made. The images or other third party material in this article are included in the article's Creative Commons licence, unless indicated otherwise in a credit line to the material. If material is not included in the article's Creative Commons licence and your intended use is not permitted by statutory regulation or exceeds the permitted use, you will need to obtain permission directly from the copyright holder. To view a copy of this licence, visit http://creativecommons.org/licenses/by/4.0/.

(C) The Author(s) 2021 